\title{
The braided Ptolemy-Thompson group is finitely presented
}

\author{
LOUIS FUNAR \\ CHRISTOPHE KAPOUDJIAN
}

\begin{abstract}
Pursuing our investigations on the relations between Thompson groups and mapping class groups, we introduce the group $T^{\sharp}$ (and its companion $T^{*}$ ) which is an extension of the Ptolemy-Thompson group $T$ by the braid group $B_{\infty}$ on infinitely many strands. We prove that $T^{\sharp}$ is a finitely presented group by constructing a complex on which it acts cocompactly with finitely presented stabilizers, and derive from it an explicit presentation. The groups $T^{\sharp}$ and $T^{*}$ are in the same relation with respect to each other as the braid groups $B_{n+1}$ and $B_{n}$, for infinitely many strands $n$. We show that both groups embed as groups of homeomorphisms of the circle and their word problem is solvable.
\end{abstract}

20F36, 57M07; 20F38, 20F05, 57N05

\section{Introduction}

The first relationships between Thompson's groups and braid groups were brought to light in the article [25] by P Greenberg and V Sergiescu, which is devoted to the construction and the homological study of extensions of Thompson's groups $F$ and $T$ by the stable braid group $B_{\infty}$. More recently, several works have contributed to the development of this subject, dealing with connections between Thompson's groups and mapping class groups of surfaces, including braid groups. Among them are two papers by the authors [22] and the second author and Sergiescu [28], which have brought out the notion of asymptotic mapping class group. The group $T^{\#}$ introduced in the present article is an asymptotic mapping class group as well.

In order to give a flavor of what an asymptotic mapping class group can be, let us briefly recall what [28] and [22] were about. One of the aims of [28] was to give a topological construction for the group $A_{T}$, defined in [25] as an extension of Thompson's group $T$ by $B_{\infty}$, and to exploit it in order to extend the Burau representation to $A_{T}$. At the same time, the article [22] was introducing $\mathcal{B}$, a universal mapping class group in genus zero, algebraically described as an extension of Thompson's group $V$, and topologically defined as a mapping class group of a sphere minus a Cantor set. Both articles [28] and [22] share a common problem, which is the following. Elements of 
Thompson's groups are not tree automorphisms, but are induced by piecewise tree automorphisms [28]. Therefore, a natural question is to find a way of lifting those elements to automorphisms of an appropriate structure. The answer proposed by [28] and [22] is to lift them to mapping classes of homeomorphisms of particular surfaces. Indeed, both groups $A_{T}$ and $\mathcal{B}$ are mapping class groups of infinite surfaces which are thickenings of suitable regular trees; the surfaces are endowed with an extra structure that must be, not globally, but only asymptotically preserved by the mapping classes hence the notion of asymptotic mapping class group. This extra structure may consist of a decomposition of the surface into pairs of pants, hexagons, hexagons with punctures, and so on.

The surface $D^{\#}$ considered for the construction of the asymptotic mapping class group $T^{\sharp}$ is the planar thickened binary tree, which is punctured along an infinite discrete subset of points. The extra structure consists of a decomposition into suitably punctured hexagons. The asymptotic mapping class group that one obtains this way is an extension $T^{\#}$ of $T$ by the group of braids $B_{\infty}$ on infinitely many strands (corresponding to the punctures). Therefore, $T^{\sharp}$ is quite similar to, but simpler than $A_{T}$.

This new group $T^{\sharp}$ seems interesting and worthy of deeper study. Compared with $\mathcal{B}$, the definition of $T^{\sharp}$ presents new features, for instance, the dependence on the extra structure is now clearly manifest. We can choose two sets of punctures leading to homeomorphic surfaces for which the associated groups are not isomorphic. We obtain that way another group $T^{*}$, which is a sort of twin brother of $T^{\sharp}$. Although $T^{\sharp}$ and $T^{*}$ share the same properties, they are different. Our main result is the following:

Theorem 0.1 The groups $T^{\sharp}$ and $T^{*}$ are nonisomorphic finitely presented groups, are extensions of the Thompson group $T$ by $B_{\infty}$, and split over the smallest Thompson group $F \subset T$. They are groups of homeomorphisms of the circle with solvable word problem. Moreover, $T^{*}$ has two generators.

We do not know if we can extend our methods to prove that the group $A_{T}$ of GreenbergSergiescu is finitely presented as well. On the other hand, we claim that the procedure of [28] applies also to $T^{\sharp}$ to extend the Burau representation of $B_{\infty}$ to $T^{\sharp}$.

We should mention that an extension $B V$ of the larger Thompson group $V$ by a pure braid group on infinitely many strands has been recently considered by $\mathrm{M}$ Brin [6; 5] and P Dehornoy [12; 13]. It constitutes the planar counterpart of the group $\mathcal{B}$, in which the Cantor surface is replaced by a disk minus a Cantor set. As a matter of fact, we have observed that $B V$ is a subgroup of $\mathcal{B}$ [22]. Since $B V$ is called the braided Thompson group, we hope to avoid any confusion by calling $T^{\#}$ the braided Ptolemy-Thompson group, insisting on its relation with the Ptolemy-Thompson group 
$T$ (see Penner [35]). The terminology used here for $T$ is expected to stress on its link with the Penner-Ptolemy groupoid. The group $T^{\#}$ is essentially different from $B V$ (and $\mathcal{B}$ ), being an extension by the whole group of braids, and not only the pure braids. Moreover, it is known that $H_{1}(B V)=0$, while $H_{1}\left(T^{\sharp}\right)$ is nontrivial (see Proposition 2.9 and Corollary 4.8).

The bulk of the paper is devoted to the proof of this theorem, with an explicit presentation for $T^{\#}$. We follow K Brown's method [7], based on the Bass-Serre theory. It consists in building a simply connected 2-dimensional complex on which $T^{\sharp}$ acts cocompactly with finitely presented stabilizers. The complex is a kind of fibration over a (conveniently reduced) Hatcher-Thurston complex of the infinite surface. The latter is a quotient of the Cayley complex of the Ptolemy-Thompson group $T$.

A similar construction was used in [22] to build up a complex for $\mathcal{B}$, but there, the problem was rather complicated because of the complexity of the Brown-Stein complex for $V$. However, the $T^{\sharp}$-complex has a specific ingredient, which makes it quite different from the $\mathcal{B}$-complex of [22]: the fiber of the fibration over the HatcherThurston complex is the Cayley complex of the braid group $B_{\infty}$. The trick here is to use a presentation of $B_{\infty}$ which exploits the homogeneity of the tree associated to the infinite surface. It is precisely provided by a general theorem due to V Sergiescu [38]. The remaining difficulty consists in dealing with countably many relations of commutations between the braid generators which occur in this presentation.

The construction of $T^{\sharp}$, as a mapping class group of a punctured infinite surface of infinite type, can be viewed also as an extension of the Dynnikov three pages representations [15], where the infinite braid group $B_{\infty}$ was realized as the commutator of a finitely presented group. Different versions of this construction are also known as the braided Houghton groups $H B_{n}$, introduced in the (unpublished) thesis [11] of F Degenhardt (see Bux [8]).

The groups $T^{*}$ and $T^{\sharp}$ generalize the diagram picture groups considered by $\mathrm{V}$ Guba and M Sapir [27] insofar as these are extensions by infinite permutation groups, rather than braid groups. Diagram groups are known to have very good properties: they are $\mathrm{FP}_{\infty}$ and have solvable conjugacy and word problems. We expect similar results for the braided Ptolemy-Thompson groups. Farley's construction [18] can be carried on in this more general setting to show that $T^{\sharp}$ acts on a CAT(0) cubical complex with braid groups as stabilizers. However, it seems more difficult in this case to obtain a subcomplex whose quotient has a finite skeleton in each dimension.

The plan of this paper is as follows. We introduce the groups $T^{\sharp}$ and $T^{*}$ as asymptotically rigid mapping class groups of infinitely punctured planar surfaces. We describe specific elements of these groups and prove that $T^{*}$ is generated by two elements and 
that the extension of $T$ is split over the smaller Thompson group $F$. This permits us to find the abelianization of $T^{*}$, to be compared with that of $T^{\sharp}$ later. We include an explanation of the close relationship between the groups $T^{\sharp}$ and $T^{*}$, which is similar to how the braid group $B_{n}$ is related to $B_{n-1}$, for infinite $n$. One shows further that both groups act on the circle by homeomorphisms. The groups $T^{\sharp}$ and $T^{*}$ have solvable word problem, by a suitable extension of Artin's solution of the word problem for braids. Section 3 is devoted to construct a simply connected complex on which $T^{\sharp}$ acts with finitely presented stabilizers. In Section 4 we show how a suitable simply connected subcomplex inherits a cocompact action. Then Brown's methods yield an explicit presentation for $T^{\sharp}$ and we sketch the proof of the necessary changes for obtaining the finite presentability of $T^{*}$. Section 5 collects some remarks and questions.

Acknowledgements The authors are thankful to D Calegari, P Dehornoy, M Rubin, V Sergiescu, B Wiest and the referees for comments and useful discussions.

\section{Infinite planar surfaces and asymptotic mapping class groups}

\subsection{Enhanced surfaces of infinite type}

The surfaces below will be oriented and all homeomorphisms considered in the sequel will be orientation-preserving, unless the opposite is explicitly stated. Actions in the sequel are left actions and the composition of maps is the usual one, namely we start composing from right to the left.

Definition 1.1 The ribbon tree $D$ is the planar surface obtained by thickening in the plane the infinite binary tree. We denote by $D^{\#}$ (respectively, $D^{*}$ ) the ribbon tree with infinitely many punctures, one puncture for each vertex (respectively, each edge) of the tree. A homeomorphism of $D^{\#}$ (respectively $D^{*}$ ) is a homeomorphism of $D$ which permutes the punctures of $D^{\#}$ (respectively $\left.D^{*}\right)$.

Definition 1.2 A rigid structure on $D, D^{\#}$ or $D^{*}$ is a decomposition into hexagons by means of a family of arcs whose endpoints are on the boundary of $D$. Each hexagon contains exactly one puncture in its interior in the case of $D^{\sharp}$, while each arc passes through a unique puncture in the case of $D^{*}$. It is assumed that these arcs are pairwise nonhomotopic in $D$, by homotopies keeping the boundary points of the arcs on the boundary of $D$. The choice of a rigid structure of reference is called the canonical 

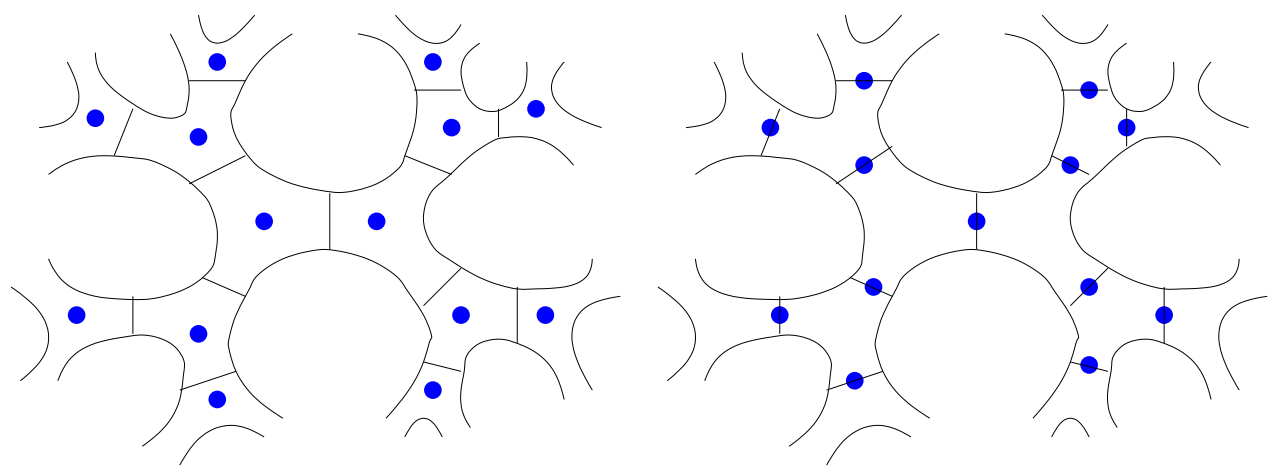

Figure 1: $D^{\sharp}$ and $D^{*}$ and with their canonical rigid structures

rigid structure. The canonical rigid structure of the ribbon tree $D$ is such that each arc of this rigid structure crosses once and transversely a unique edge of the tree. The canonical rigid structures on $D^{\sharp}$ and $D^{*}$ are assumed to coincide with the canonical rigid structure of $D$ when forgetting the punctures. See Figure 1.

Notation 1.1 Let $\diamond$ stand for \#, * or the vacuum. The set of isotopy classes of rigid structures on $D^{\diamond}$ will be denoted $\mathfrak{R}^{\diamond}$. The canonical rigid structure of $D^{\diamond}$ will be denoted $\mathfrak{r}^{\diamond}$.

\subsection{Asymptotic mapping class groups}

Definition 1.3 (1) Let $D^{\diamond}$ denote $D, D^{\sharp}$ or $D^{*}$. A planar subsurface of $D^{\diamond}$ is admissible if it is a connected finite union of hexagons belonging to the canonical rigid structure $\mathfrak{r}^{\diamond}$. The frontier of an admissible surface is the union of the arcs contained in the boundary. The remaining arcs will be called separating arcs.

(2) Let $\varphi$ be a homeomorphism of $D^{\diamond}$. One says that $\varphi$ is asymptotically rigid if the following conditions are fulfilled:

- There exists an admissible subsurface $\Sigma \subset D^{\diamond}$ such that $\varphi(\Sigma)$ is also admissible.

- The complement $D^{\diamond}-\Sigma$ is a union of $n$ infinite surfaces. Then the restriction $\varphi: D^{\diamond}-\Sigma \rightarrow D^{\diamond}-\varphi(\Sigma)$ is rigid, meaning that it respects the canonical rigid structures in the complements of the compact subsurfaces, mapping hexagons into hexagons. Such a surface $\Sigma$ is called a support for $\varphi$.

One denotes by $T, T^{\sharp}$ and $T^{*}$ the group of isotopy classes of asymptotically rigid homeomorphisms of $D, D^{\sharp}$ and $D^{*}$, respectively. 
Remark 1.2 There exists a cyclic order on the frontier arcs of an admissible subsurface induced by the planarity. An asymptotically rigid homeomorphism necessarily preserves the cyclic order of the frontier for any admissible subsurface.

\subsection{Ptolemy-Thompson group $T$ as a mapping class group}

The mapping class group $T$ is isomorphic to the Thompson group which is commonly denoted $T$. This fact has been widely developed in [28] and [22]. We consider the following elements of $T$, defined as mapping classes of asymptotically rigid homeomorphisms:

- The support of the element $\beta$ is the central hexagon on the figure below. Further, $\beta$ acts as the counterclockwise rotation of order three which permutes the three branches of the ribbon tree issued from the hexagon.

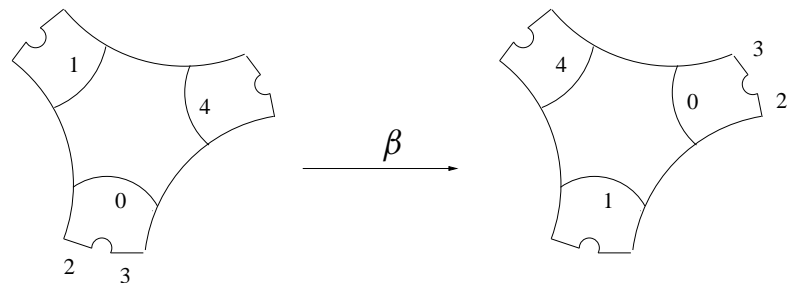

In fact, $\beta$ is globally rigid.

- The support of $\alpha$ is the union of two adjacent hexagons, one of them being the support of $\beta$. Then $\alpha$ rotates counterclockwise the support of angle $\frac{\pi}{2}$, by permuting the four branches of the ribbon tree issued from the support.

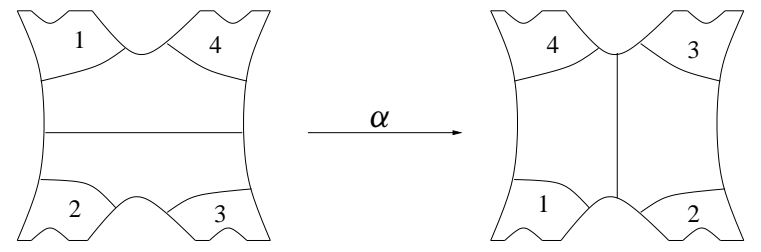

Note that $\alpha$ is not globally rigid, but $\alpha^{2}$ is.

Proposition 1.3 The group $T$ has the following presentation with generators $\alpha$ and $\beta$ and relations

$$
\begin{gathered}
\alpha^{4}=\beta^{3}=1 \\
{\left[\beta \alpha \beta, \alpha^{2} \beta \alpha \beta \alpha^{2}\right]=1} \\
{\left[\beta \alpha \beta, \alpha^{2} \beta^{2} \alpha^{2} \beta \alpha \beta \alpha^{2} \beta \alpha^{2}\right]=1} \\
(\beta \alpha)^{5}=1 .
\end{gathered}
$$


Proof This result is due to Lochak and Schneps [30], but there is a typo in their statement, which is corrected above.

Remark 1.4 If we set $A=\beta \alpha^{2}, B=\beta^{2} \alpha$ and $C=\beta^{2}$ then we obtain the generators $A, B, C$ of the group $T$, considered by Cannon, Floyd and Parry [10]. Then the two commutativity relations above are equivalent to:

$$
\left[A B^{-1}, A^{-1} B A\right]=1, \quad\left[A B^{-1}, A^{-2} B A^{2}\right]=1
$$

The presentation of $T$ in terms of the generators $A, B, C$ consists of the two relations above with four more relations to be added:

$$
C^{3}=1, C=B A^{-1} C B, C A=\left(A^{-1} C B\right)^{2},\left(A^{-1} C B\right)\left(A^{-1} B A\right)=B\left(A^{-2} C B^{2}\right)
$$

Remark 1.5 The subgroup of $T$ generated by the elements $A$ and $B$ is the Thompson group $F$, as it is obvious from [10]. Moreover, the group $F$ has the presentation

$$
F=\left\langle A, B ;\left[A B^{-1}, A^{-1} B A\right]=1,\left[A B^{-1}, A^{-2} B A^{2}\right]=1\right\rangle
$$

Consequently, the inclusion $F \rightarrow T$ sends $A$ to $A$ and $B$ to $B$.

\section{The braided Ptolemy-Thompson groups $T^{\sharp}$ and $T^{*}$}

\section{1 $T^{\sharp}$ and $T^{*}$ as extensions of the Thompson group $T$}

We write $D$ (respectively $D^{\sharp}$ and $D^{*}$ ) as an ascending union $\bigcup_{n} D_{n}$, where $D_{0}$ is the support of $\beta$, and $D_{n+1}$ is obtained by adding to $D_{n}$ a new hexagon from the canonical rigid structure, along each arc of the frontier.

Let the symbol $\diamond$ denote either ${ }^{\#}$ or *. The Artin braid groups $B\left[D_{n}^{\diamond}\right]$ associated to the punctures on $D_{n}^{\diamond}$ form an inductive system induced by the inclusions $D_{n}^{\diamond} \subset D_{n+1}^{\diamond}$, whose limit $B\left[D^{\diamond}\right]=\lim _{n \rightarrow \infty} B\left[D_{n}^{\diamond}\right]$ can be identified with the group of compactly supported braids on $D$, where the base points of the strands are the punctures of $D^{\diamond}$.

Remark 2.1 The group $B\left[D^{\diamond}\right]$ is isomorphic to the stable braid group $B_{\infty}$, where $B_{\infty}$ is the inductive limit coming from the inclusions $\sigma_{i} \in B_{n} \mapsto \sigma_{i} \in B_{n+1}$, where $\sigma_{i}$ $(1 \leq i \leq n-1)$ denotes a standard Artin generator. This can be proven by observing first that the embedding of $D^{\diamond}$ into the Euclidean plane $P$ induces an isomorphism of $B\left[D^{\diamond}\right]$ with the group of isotopy classes of compactly supported homeomorphisms of the punctured plane. Since the set of punctures is discrete in $P$, one may construct a homeomorphism of $P$ which maps the punctures on the points of coordinates $(i, 0)$, $i \in \mathbb{N}^{*}$ (after a choice of a framing). By conjugation, this homeomorphism induces an isomorphism between $B\left[D^{\diamond}\right]$ and $B_{\infty}$. 
Proposition 2.2 Let the symbol ${ }^{\diamond}$ denote either ${ }^{\sharp}$ or * . We have an exact sequence:

$$
1 \rightarrow B\left[D^{\diamond}\right] \rightarrow T^{\diamond} \rightarrow T \rightarrow 1
$$

Proof Thompson's group $T$ is viewed here as the group of isotopy classes of asymptotically rigid homeomorphisms of $D$ (without punctures) up to isotopy. Thus, the epimorphism $T^{\diamond} \rightarrow T$ is induced by forgetting the punctures. Now let $\varphi$ be an asymptotically rigid homeomorphism of $D^{\diamond}$ whose image in $T$ is trivial. This implies that outside an admissible subsurface, $\varphi$ is isotopic to identity. Without changing the class of $\varphi$ in $T^{\diamond}$, we may assume that outside this subsurface, $\varphi$ is identity. Therefore, there exists a compactly supported isotopy $\varphi_{t}$ among homeomorphisms of $D$ which joins $\varphi$ to identity, whose support is an admissible subsurface. Further the class of the homeomorphism $\varphi$ on the punctured support is completely encoded by a braid, and a picture showing the trajectory of the punctures during the isotopy. Then the class of $\varphi$ is the image of a braid from a some suitable $B\left[D_{n}^{\diamond}\right]$ into $T^{\diamond}$. This means that the kernel above is $B\left[D^{\diamond}\right]$.

\section{2 $T^{*}$ is generated by two elements}

Thompson's groups and their generalizations considered by Higman are generated by two elements [32]. It is known that mapping class groups of closed surfaces of genus at least one are also generated by two elements. We will prove here that the same holds for the group $T^{*}$.

Specific elements Let us consider the following elements of $T^{\#}$ and $T^{*}$ :

- The support of the element $\beta^{*}$ of $T^{*}$ (respectively $\beta^{\sharp}$ of $T^{\sharp}$ ) is the central hexagon. Further $\beta^{*}$ and $\beta^{\sharp}$ act as the counterclockwise rotation of order three which permutes cyclically the punctures. One has $\beta^{*^{3}}=1$ and $\beta^{\sharp 3}=1$. See Figure 2.

- The support of the element $\alpha^{*}$ of $T^{*}$ (respectively $\alpha^{\sharp}$ of $T^{\sharp}$ ) is the union of two adjacent hexagons, one of them being the support of $\beta^{*}$ and $\beta^{\sharp}$. Then $\alpha^{*}$ (respectively $\alpha^{\sharp}$ ) rotates counterclockwise the support of angle $\frac{\pi}{2}$, by keeping fixed the central puncture (respectively the two punctures of the adjacent hexagons). See Figure 3. One has $\alpha^{* 4}=1$ while $\alpha^{\sharp 4}=\sigma^{2}$, where $\sigma$ denotes the braid that permutes the puncture 0 and 3; see Definition 2.1 below. 

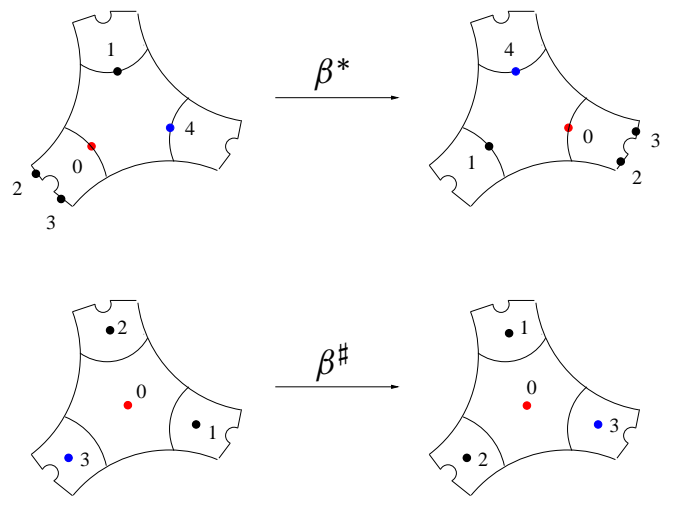

Figure 2
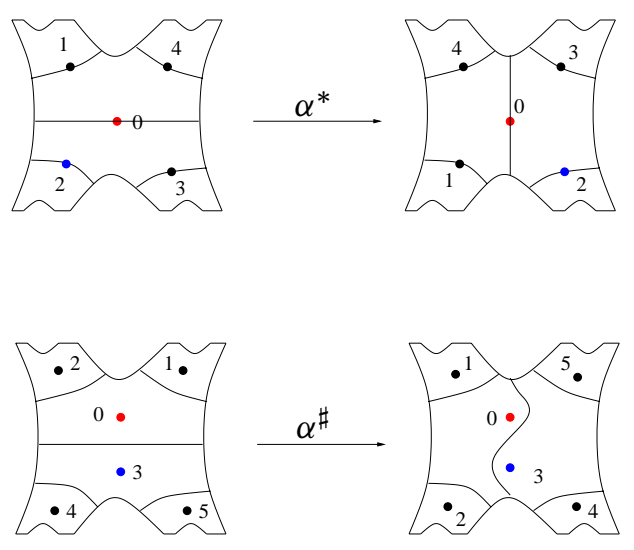

Figure 3

Definition 2.1 Let $e$ be a simple arc in $D^{\sharp}$ or $D^{*}$ which connects two punctures. We associate a braiding $\sigma_{e} \in B_{\infty}$ to $e$ by considering the homeomorphism that moves clockwise the punctures at the endpoints of the edge $e$ in a small neighborhood of the edge, in order to interchange their positions. This means that, if $\gamma$ is an arc transverse to $e$, then the braiding $\sigma_{e}$ moves $\gamma$ on the left when it approaches $e$. Such a braiding will be called positive, while $\sigma_{e}^{-1}$ is negative. See Figure 4.

Remark 2.3 The subgroup of $T$ generated by $\alpha^{2}$ and $\beta$, as well as the subgroup of $T^{*}$ generated by $\alpha^{* 2}$ and $\beta^{*}$, are isomorphic to $\operatorname{PSL}(2, \mathbb{Z})$, viewed as the group of orientation-preserving automorphisms of the binary planar tree of the ribbon surface 


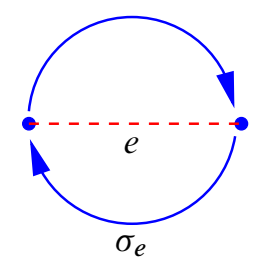

Figure 4

$D$. In the same way, the subgroup of $T^{\sharp}$ generated by $a=\alpha^{\sharp 2} \sigma^{-1}$ (which is of order 2) and $\beta^{\sharp}$ is isomorphic to $\operatorname{PSL}(2, \mathbb{Z})$.

Theorem 2.4 $T^{*}$ is generated by $\alpha^{*}$ and $\beta^{*}$.

Proof Let us denote by $T^{\prime}$ the subgroup of $T^{*}$ generated by $\alpha^{*}, \beta^{*}$.

Lemma 2.5 The restriction to $T^{\prime}$ of the projection map $T^{*} \rightarrow T$ is surjective.

Proof This maps sends $\alpha^{*}$ to $\alpha$ and $\beta^{*}$ to $\beta$. According to Proposition 1.3, $\alpha$ and $\beta$ generate $T$, thus the claim.

Let now $\iota: B_{\infty} \rightarrow T^{*}$ be the natural inclusion. Since $T^{*}$ is an extension of $T$ by $B_{\infty}$, it suffices now to show that $\iota\left(B_{\infty}\right) \subset T^{\prime}$. This will be done in two steps. First we show that a specific braid generator lies in $T^{\prime}$ and next we use the conjugation action to prove that all braid generators lay within $T^{\prime}$. The first step proceeds as follows.

Lemma 2.6 The braid generator $\sigma_{[04]}$ associated to the edge joining the punctures numbered 0 and 4 has image

$$
\iota\left(\sigma_{[04]}\right)=\left(\beta^{*} \alpha^{*}\right)^{5}
$$

Proof We claim that the action of $\left(\beta^{*} \alpha^{*}\right)^{5}$ on the standard rigid structure of the ribbon tree is the following one:

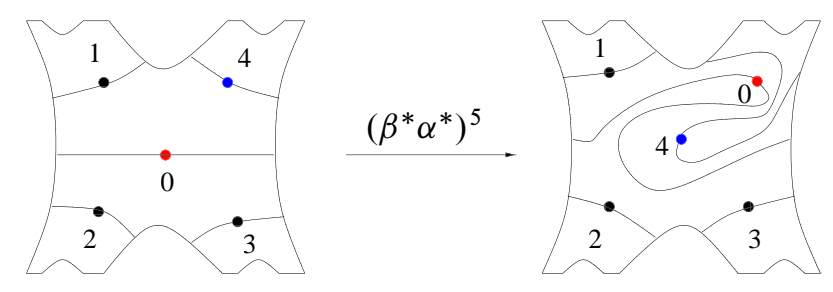



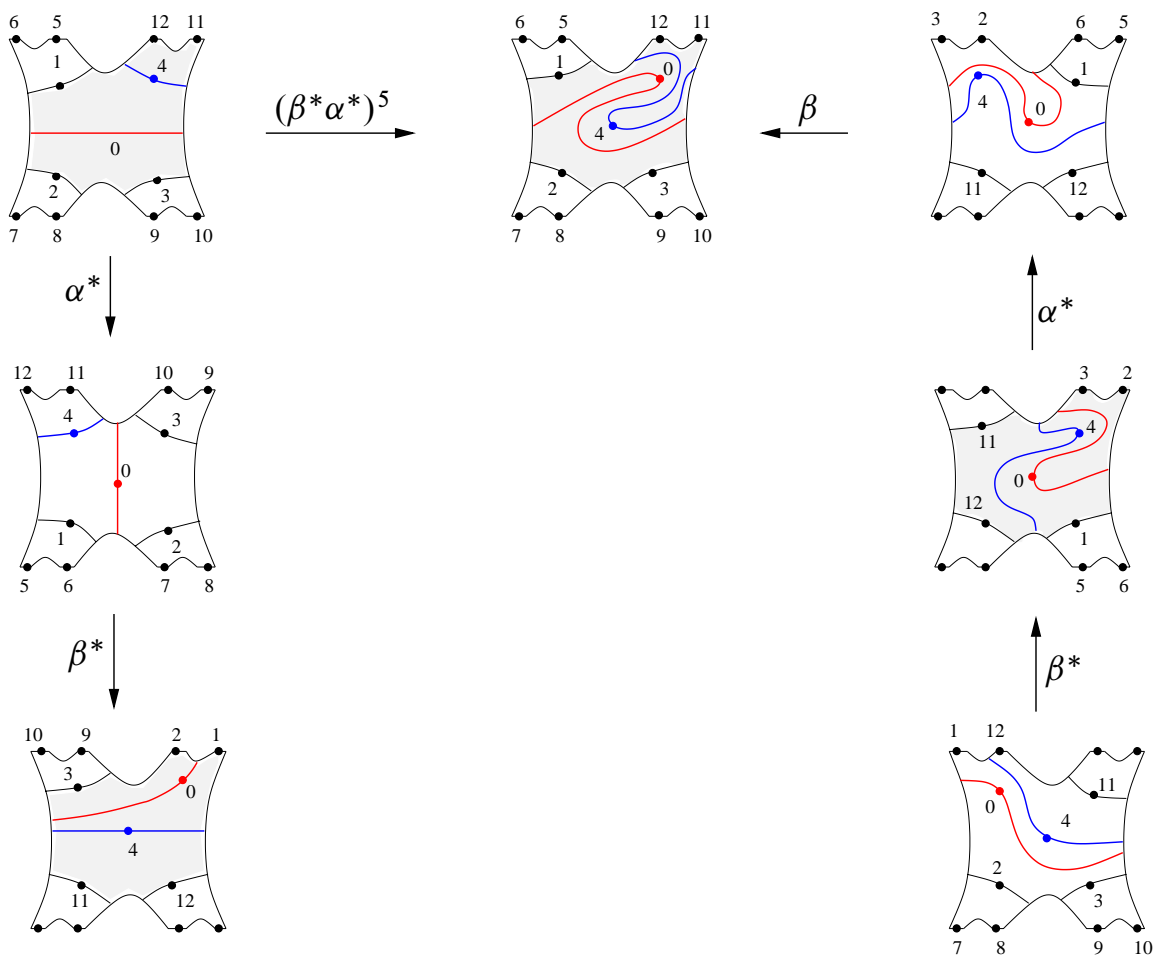

$\alpha^{*}$
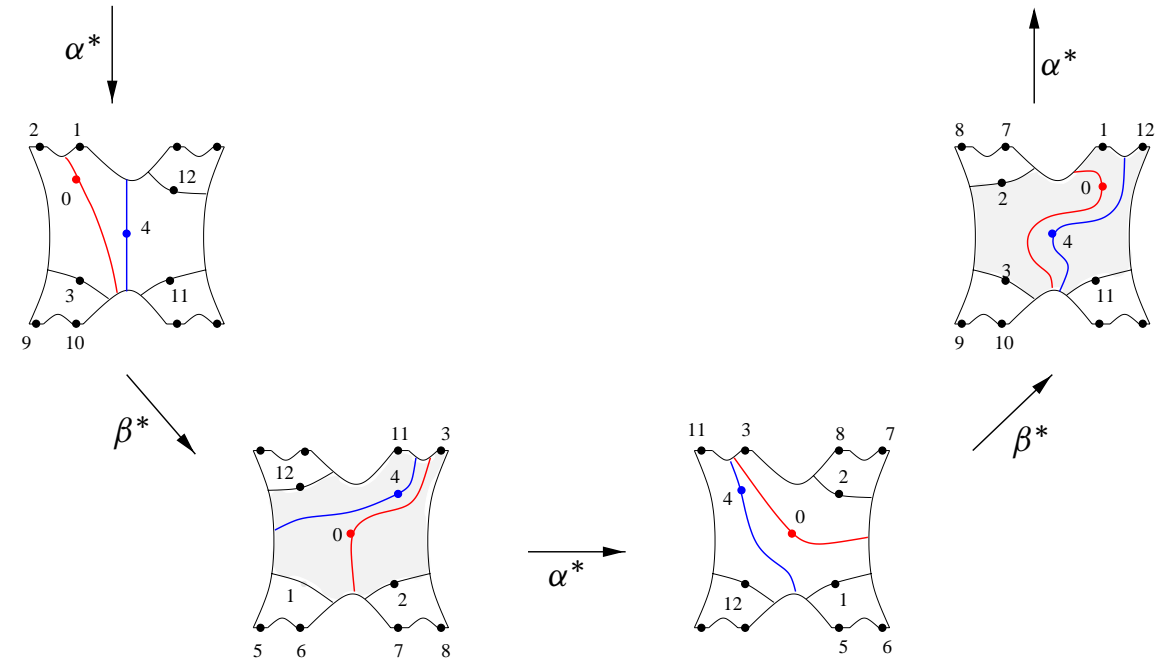

Figure 5: Proof of $\iota\left(\sigma_{[04]}\right)=\left(\beta^{*} \alpha^{*}\right)^{5}$

This follows from the explicit picture calculations in Figure 5. Remark that $\beta^{*} \alpha^{*}$ acts as an order five rotation whose support is the dashed decagon in the picture. 
In particular, the action of $\left(\beta^{*} \alpha^{*}\right)^{5}$ can be identified with the action of the mapping class $\sigma_{[04]}$ on rigid structures. Then the action of $\left(\beta^{*} \alpha^{*}\right)^{5}$ coincides with the natural action of $\sigma_{[04]} \in B_{\infty}$ on the arcs in the punctured surface. In meantime the configuration of arcs coming from a rigid structure (up to isotopy) uniquely determines the element of $B_{\infty}$, and so $\iota\left(\left(\beta^{*} \alpha^{*}\right)^{5}\right)=\sigma_{[04]}$.

The end of the proof is now as follows. For each hexagon of $D^{*}$, consider the three arcs which connect the punctures to each other, and intersect only at the punctures. Let $\mathcal{E}$ be the set of all such arcs associated to $D^{*}$. The subgroup of $T^{*}$ generated by $\alpha^{* 2}$ and $\beta^{*}$ acts transitively on $\mathcal{E}$ as the group $\operatorname{PSL}(2, \mathbb{Z})$. Therefore, for each $e \in \mathcal{E}$, there exists a word $w$ in $\alpha^{* 2}$ and $\beta^{*}$ such that $w\left(e_{[04]}\right)=e$. Then $w \sigma_{[04]} w^{-1}=\sigma_{e}$. Consequently, each $\sigma_{e}$ belongs to $T^{\prime}$. Since the group $B_{\infty}$ is generated by the braidings $\sigma_{e}$ when $e$ runs over $\mathcal{E}, T^{\prime}$ contains $\iota\left(B_{\infty}\right)$.

Remark 2.7 The union of all edges of $\mathcal{E}$ is a graph, which is dual to the binary tree (of $D$ or $D^{\sharp}$ ). It will be called the graph of $D^{*}$, see Figure 6. A general theorem due to $\mathrm{V}$ Sergiescu [38] implies that $B_{\infty}$ is generated by $\left\{\sigma_{e}, e \in \mathcal{E}\right\}$. The relations holding between these generators are made explicit in [38]. This approach was later generalized by Birman, Ko and Lee [4].
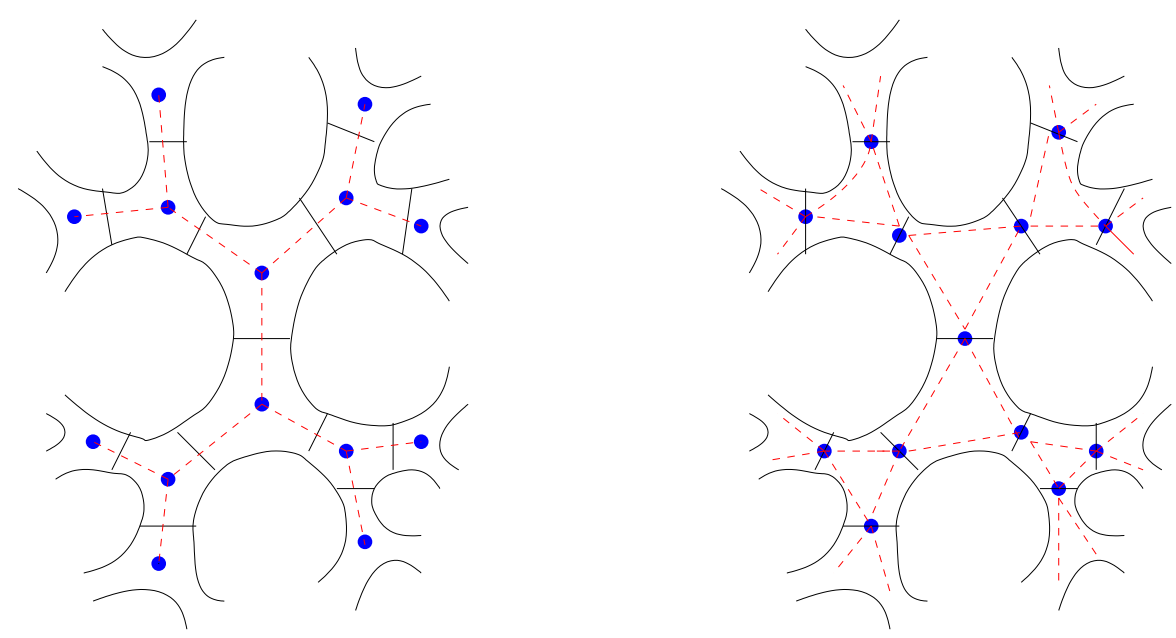

Figure 6: Tree of $D^{\sharp}$ and graph of $D^{*}$

Proposition 2.8 The exact sequence associated to $T^{*}$ is split over $F \subset T$. 
Proof We define the section $F \rightarrow T^{*}$ by sending the generators $A$ and $B$ of $F$ onto $\beta^{*} \alpha^{* 2}$ and $\beta^{* 2} \alpha^{*}$, respectively, which are the same words as those defining the inclusion into $T$. It is worthy to visualize the actions of the two elements, which by notation abuse we will keep denoting by $A$ and $B$ :
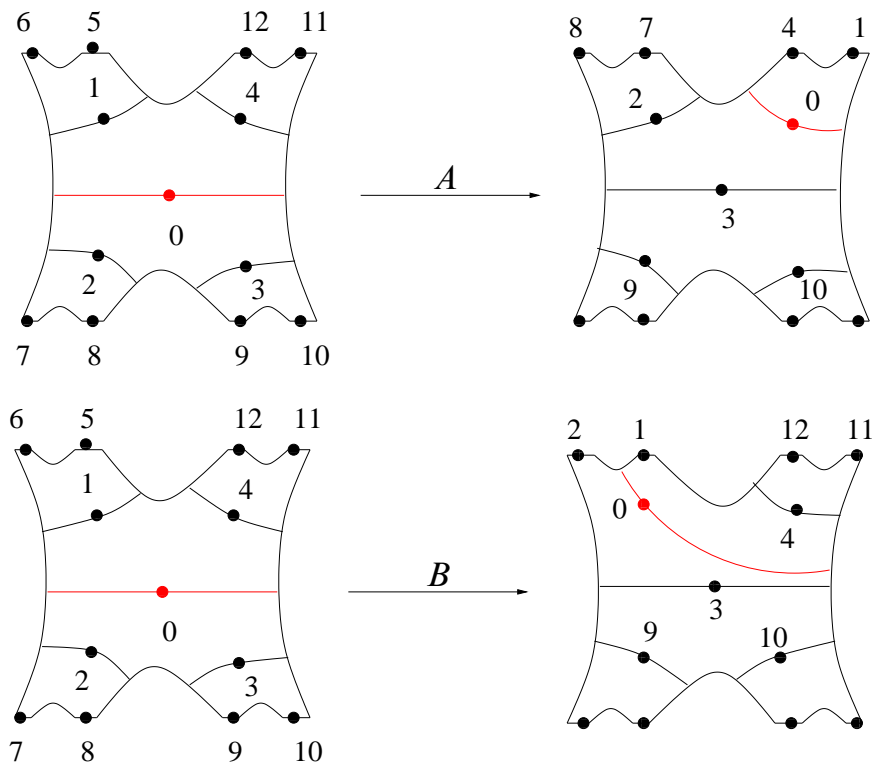

Now it is not difficult to compute $A B^{-1}$ and $A^{-1} B A$ :
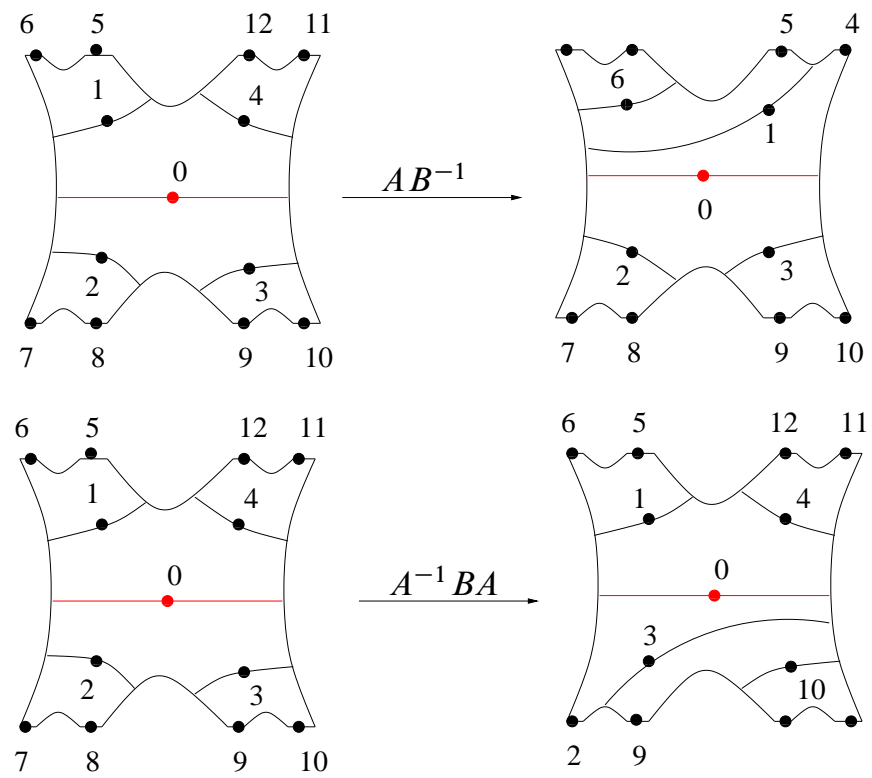

Geometry 8 Topology, Volume 12 (2008) 
Observe that $A B^{-1}$ acts effectively only on the upper half-plane determined by the horizontal line through the puncture labeled 0 , while $A^{-1} B A$ acts effectively only on the lower half-plane determined by the same line.

Thus these two mapping classes come from homeomorphisms with disjoint supports and thus they commute within $T^{*}$. The same holds for $A B^{-1}$ and $A^{-2} B A^{2}$. In particular, the relations which define the group $F$ are satisfied by the images of $A$ and $B$ in $T^{*}$ and thus we obtained a section.

\subsection{The abelianization $H_{1}\left(T^{*}\right)$}

Proposition 2.9 The abelianization $H_{1}\left(T^{*}\right)$ is isomorphic to $\mathbb{Z} / 12 \mathbb{Z}$.

Proof We know that $T^{*}$ fits into an exact sequence:

$$
1 \rightarrow B_{\infty} \rightarrow T^{*} \rightarrow T \rightarrow 1
$$

The group $B_{\infty}$ is the group of braids associated to the punctures of $D^{*}$. We will consider the Sergiescu presentation of $B_{\infty}$ associated to the graph of $D^{*}$; see Remark 2.7. Hall's lemma (see Robinson [37]) provides an infinite presentation for $T^{*}$ which puts together the presentations of $T$ and $B_{\infty}$.

The relations satisfied by the generators $\alpha, \beta$ of $T$ have the following lifts in $T^{*}$ :

$$
\begin{gathered}
\alpha^{* 4}=\beta^{* 3}=1 \\
{\left[\beta^{*} \alpha^{*} \beta^{*}, \alpha^{* 2} \beta^{*} \alpha^{*} \beta^{*} \alpha^{* 2}\right]=1} \\
{\left[\beta^{*} \alpha^{*} \beta^{*}, \alpha^{* 2} \beta^{* 2} \alpha^{* 2} \beta^{*} \alpha^{*} \beta^{*} \alpha^{* 2} \beta^{*} \alpha^{* 2}\right]=1} \\
\left(\beta^{*} \alpha^{*}\right)^{5}=\sigma_{[04]}
\end{gathered}
$$

where $\sigma_{[04]}$ is the braid generator considered above (see Lemma 2.6). The first two relations are obvious. The next two are actually expressing the fact that the defining relations in $F$ are satisfied by $A=\beta^{*} \alpha^{* 2}$ and $B=\beta^{* 2} \alpha^{*}$, and thus represent a restatement of Proposition 2.8. The last relation is that from Lemma 2.6.

All relations involving the braids come from Sergiescu's relations, while the remaining relations in $T^{*}$ are conjugacy relations stating that $B_{\infty}$ is normal inside $T^{*}$. Thus the abelianization $H_{1}\left(T^{\sharp}\right)$ is generated by the classes of $\alpha^{*}$ and $\beta^{*}$ which are constrained to be of order 4 and 3, respectively. The claim follows.

The explicit presentation of $T^{\sharp}$ given in the section 4 will show that $H_{1}\left(T^{\sharp}\right)=\mathbb{Z} / 6 \mathbb{Z}$ and thus $T^{\sharp}$ and $T^{*}$ are not isomorphic (see Corollary 4.8). 


\section{$2.4 T^{\sharp} \operatorname{versus} T^{*}$}

We will show below that $T^{\sharp}$ is related to $T^{*}$ in the same way as the braid group $B_{n}$ is related to $B_{n-1}$, for infinite $n$. Roughly speaking, one obtains $T^{*}$ by considering the mapping classes of $T^{\#}$ associated to those homeomorphisms fixing one specific puncture of $D^{\sharp}$, and by viewing them as mapping classes of $D^{\sharp}$ union that puncture.

Specifically, denote by $T_{*}^{\#}$ the subgroup of $T^{\#}$ formed by those homeomorphism classes that keep fixed the point $q$ labeled 3 in the picture of $D^{\sharp}$ (see Figure 2), ie the center of the hexagon adjacent to and located below the support of $\beta^{\sharp}$.

Let $B_{\infty, 1} \subset B_{\infty}$ denote the subgroup of braids that keep fixed the puncture $q$, and $B_{\infty-1}$ denote the infinite braid on the punctures of $D^{\sharp} \cup\{q\}$. There is an obvious projection map $B_{\infty, 1} \rightarrow B_{\infty-1}$ that consists in deleting the strand over $q$.

Proposition 2.10 We have a commutative diagram with exact lines and columns:

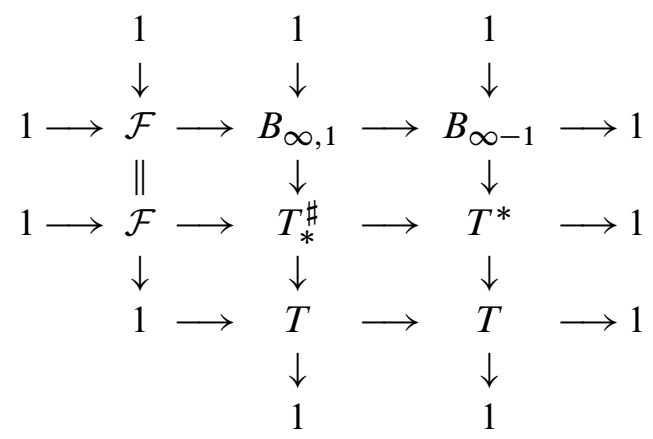

where $\mathcal{F}$ is a free group, normally generated by $\sigma^{2}=\alpha^{\sharp 4}$ as a subgroup of $T_{*}^{\sharp}$.

Proof We define a homeomorphism $\varphi$ of $D^{\sharp} \cup\{q\}$ onto $D^{*}$ that extends to rigid structures, as follows. Suppose that the locations of punctures are the same in both models $D^{\#}$ and $D^{*}$ and just the position of the separating arcs in the rigid structures change.

Each hexagon $H_{i}$ of $D^{\sharp}$ contains in its interior the puncture labeled $i$. Delete the puncture $q$ and get therefore one hexagon $H_{q}$ containing no puncture, which we call the empty hexagon. One joins the hexagon $H_{i}$ to the empty one by a geodesic (ie minimal length) path through adjacent hexagons. In particular, consecutive hexagons in the path have precisely one edge in common and the sequence of common edges has no repetitions. Then each hexagon $H_{i}$ (different from the empty one) has one distinguished separating arc $\gamma_{i}$, namely the first term of the sequence of common edges 
associated to the path. In other terms, $\gamma_{i}$ is that separating arc of $H_{i}$ which is closest to the empty hexagon.

Let $\delta_{i}$ be a simple arc embedded in $H_{i}$ joining the puncture $i$ to the midpoint of $\gamma_{i}$. A small enough thickening of $\delta_{i} \cup \gamma_{i}$ in $D^{\#}$ is a curvilinear quadrilateral $Q_{i}$ having two arcs in the boundary of $D^{\sharp}$ and two arcs in the interior of $D^{\sharp}$. One of the latter is an arc parallel to $\gamma_{i}$ and the other one is what we call $\gamma_{i}^{-}$. Let $\gamma_{i}^{+}$be an arc in $Q_{i}$ which is parallel to $\gamma_{i}^{-}$and passes through the puncture $i$. There is a homeomorphism $\varphi_{i}$ of $Q_{i}$, inducing the identity on its boundary, which maps $\gamma_{i}$ onto $\gamma_{i}^{+}$. Let $\varphi$ be the homeomorphism of $D$ which restricts to $\varphi_{i}$ on each $Q_{i}$, and is the identity elsewhere. It may be viewed as a homeomorphism from $D^{\sharp} \cup\{q\}$ onto $D^{*}$, which respects the rigid structures.

Conjugation by $\varphi$ induces a surjection at the mapping class groups level $\Phi: T_{*}^{\sharp} \rightarrow T^{*}$. Specifically, if $[g] \in T_{*}^{\sharp}$, where $g$ is a homeomorphism of $D^{\sharp} \cup\{q\}$ and $[g]$ the associated mapping class, then $\Phi([g]) \in T^{*}$ is $\left[\varphi \circ g \circ \varphi^{-1}\right]$.

A standard argument identifies the kernel $\mathcal{F}$ of the map $\Phi$ with the fundamental group $\pi_{1}\left(D^{\sharp} \cup\{q\}, q\right)$ based at $q$. In particular, $\mathcal{F}$ is an infinitely generated free group and $\sigma^{2} \in \mathcal{F}$. Since the $T_{*}^{\sharp}$-action on classes of closed loops that encircle a puncture of $D^{\sharp} \cup\{q\}$ is transitive, we derive that the normal closure of $\sigma^{2}$ is the fundamental group $\pi_{1}\left(D^{\sharp} \cup\{q\}, q\right)$, hence the whole $\mathcal{F}$, as claimed. The remaining part of the commutative diagram above follows from this description of $\mathcal{F}$.

The group $B_{\infty, 1}$ is generated by the braid generators $\sigma_{[a b]}$ with $a, b \neq 3$, and conjugates of $\sigma^{2}$. Here $\sigma_{[a b]}$ is the braid generator associated to the edge whose vertices are labeled $a$ and $b$ and $\sigma$ denotes $\sigma_{[03]} \in T^{\sharp}$. Thus,

$$
\Phi\left(\sigma_{[a b]}\right)=\sigma_{[\varphi(a) \varphi(b)]}, \quad \Phi\left(\sigma^{2}\right)=1 .
$$

Moreover, by direct inspection we find that

$$
\Phi\left(\alpha^{\sharp}\right)=\alpha^{*}, \Phi\left(\sigma \beta^{\sharp} \sigma^{-1}\right)=\beta^{*} .
$$

The close relation between the two groups permits to derive a splitting result similar to that from Proposition 2.8:

Proposition 2.11 The exact sequence associated to $T^{\sharp}$ is split over $F \subset T$.

Proof Recall from the proof of Proposition 2.8 that $F \subset T^{*}$ is generated by $A=$ $\beta^{*} \alpha^{* 2}$ and $B=\beta^{* 2} \alpha^{*}$. We claim now that the map defined by

$$
\iota(A)=\sigma \beta^{\sharp} \sigma^{-1} \alpha^{\sharp 2}, \iota(B)=\sigma \beta^{\sharp 2} \sigma^{-1} \alpha^{\sharp}
$$


extends to an injective homomorphism $\iota: F \rightarrow T_{*}^{\sharp} \subset T^{\#}$ which is a section for $\Phi$. In order to prove it, one can either verify directly by picture computations that the relations in $F$ are satisfied, or else anticipate the presentation of $T^{\sharp}$ from Section 4 and observe that

$$
\begin{gathered}
\iota\left(A B^{-1}\right)=\beta^{\sharp} \alpha^{\sharp} \beta^{\sharp}, \quad \iota\left(A^{-1} B A\right)=\alpha^{\sharp 2} \sigma^{-1} \beta^{\sharp} \alpha^{\sharp} \beta^{\sharp} \alpha^{\sharp 2} \sigma^{-1} \\
\iota\left(A^{-2} B A^{2}\right)=\alpha^{\sharp 2} \sigma^{-1} \beta^{\sharp 2} \alpha^{\sharp 2} \sigma^{-1} \beta^{\sharp} \alpha^{\sharp} \beta^{\sharp} \alpha^{\sharp 2} \sigma^{-1} \beta^{\sharp} \alpha^{\sharp 2} \sigma^{-1}
\end{gathered}
$$

and thus relations in $F$ correspond to relations (5) and (6) from Theorem 4.7.

Remark 2.12 It seems that the surjection $T_{*}^{\sharp} \rightarrow T^{*}$ is not split, although it is so over the subgroups $B_{\infty-1}$ and over $F$.

\section{5 $T^{\sharp}$ is a group of homeomorphisms of $S^{1}$}

Proposition 2.13 Let the symbol ${ }^{\diamond}$ denote either ${ }^{\sharp}$ or * . There exists an embedding

$$
T^{\diamond} \rightarrow \mathrm{Homeo}_{+}\left(S^{1}\right)
$$

so that $T^{\diamond}$ acts faithfully on the circle.

Proof It is known (see Ghys and Sergiescu [24]) that $T$ can be embedded as the subgroup of piecewise linear homeomorphisms of $S^{1}=\mathbb{R} / \mathbb{Z}$ which have dyadic break points and dyadic derivatives (where defined). This implies that $T$ admits a circular order. Furthermore, the group $B_{\infty}$, as all finite type braid groups, is left orderable (see eg Dehornoy, Dynnikov, Rolfsen and Wiest [14, Proposition 9.2.7]). By using the exact sequence

$$
1 \rightarrow B_{\infty} \rightarrow T^{\diamond} \rightarrow T \rightarrow 1
$$

we define a circular order on $T^{\diamond}$, as follows. Let $\pi: T^{\diamond} \rightarrow T$ the projection and $x, y, z$ be three elements of $T^{\diamond}$.

(1) If $\pi(x), \pi(y), \pi(z)$ are distinct then their order in $T^{\sharp}$ is that of their images in $T$.

(2) If $\pi(x)=\pi(y) \neq \pi(z)$, then $x^{-1} y \in B_{\infty}$ which is left orderable. If $x^{-1} y>1$ then $x, y, z$ are positively oriented, otherwise it is negatively oriented.

(3) If $\pi(x)=\pi(y)=\pi(z)$ then $x^{-1} y, x^{-1} z \in B_{\infty}$. Assume that $1, x^{-1} y, x^{-1} z$ are totally ordered using the order in $B_{\infty}$. Then the corresponding $x, y, z$ are positively oriented in $T^{\diamond}$.

This yields a circular order on $T^{\diamond}$ and thus there exists an embedding $T^{\diamond} \hookrightarrow$ Homeo $_{+}\left(S^{1}\right)$. From [9, Theorem 2.2.15], there is a faithful $T^{\diamond}$-action on $S^{1}$. See Calegari [9] for more details about circular orders and related questions. 
Any circularly ordered group $G$ has an embedding $G \rightarrow \operatorname{Homeo}_{+}\left(S^{1}\right)$. A construction due to Ghys and Thurston yields a bounded cocycle $e$ on $G$ which is the pullback of the Euler cocycle on $\mathrm{Homeo}_{+}\left(S^{1}\right)$, and which takes only the values 0 and 1 . This defines an Euler class $[e] \in H^{2}(G)$, which is the Euler class of the circular order. It is known that $[e]=0$ only if $G$ is left ordered. Moreover, in the case of $T^{\diamond}$ with its circular ordered defined above, one knows that $T^{\diamond}$ cannot be left ordered since it has torsion. This proves the following:

Proposition 2.14 The Euler class $\left[e_{T} \diamond\right] \in H^{2}\left(T^{\diamond}\right)$ is a nontrivial bounded class, whose restriction to $B_{\infty}$ is trivial.

Moreover, this Euler class could be also described in cohomological terms. Set $e_{T}$ for the Euler cocycle on the group $T$, namely the cocycle inherited from its natural embedding into $\mathrm{Homeo}_{+}\left(S^{1}\right)$.

Proposition 2.15 The class $\left[e_{T}\right] \in H^{2}\left(T^{\diamond}, \mathbb{Q}\right)$ is the pull-back $\pi^{*}\left[e_{T}\right]$ by the projection $\pi: T^{\diamond} \rightarrow T$.

Proof According to a result due to Ghys, Jekel, and Thurston we have

$$
[c]=2[e]
$$

where $c$ is the order cocycle defined by Thurston (see Construction 2.3.4 of [9]) and $e$ is the Euler cocycle of a circularly ordered group. If the group $G$ acts faithfully on $S^{1}$ let us choose a point $a \in S^{1}$ with trivial stabilizer. Recall that $c$ is defined (as a homogeneous cocycle) by means of

$$
c\left(g_{0}: g_{1}: g_{2}\right)=\left\{\begin{array}{cll}
1 & \text { if }\left(g_{0}(a), g_{1}(a), g_{2}(a)\right) & \text { is positively oriented } \\
-1 & \text { if }\left(g_{0}(a), g_{1}(a), g_{2}(a)\right) & \text { is negatively oriented } \\
0 & \text { if }\left(g_{0}(a), g_{1}(a), g_{2}(a)\right) & \text { is degenerate }
\end{array}\right.
$$

We claim that $\pi^{*}\left(\left[c_{T}\right]\right)=\left[c_{T} \diamond\right]$. More generally, if $p: G \rightarrow H$ is a monotone homomorphism with left orderable kernel between circularly orderable groups $G$ and $H$ then $p^{*}\left[c_{H}\right]=\left[c_{G}\right]$.

In fact, we have

$$
c_{G}-p^{*} c_{H}=\partial w
$$

where $w$ is the following 1-cocycle (in homogeneous coordinates):

$$
w\left(g_{0}: g_{1}\right)=\left\{\begin{array}{c}
0 \text { if } p\left(g_{0}\right) \neq p\left(g_{1}\right) \\
1 \text { if } p\left(g_{0}\right)=p\left(g_{1}\right) \text { and } g_{0}^{-1} g_{1}<1 \\
-1 \text { if } p\left(g_{0}\right)=p\left(g_{1}\right) \text { and } g_{0}^{-1} g_{1}>1 \\
0 \text { if } \quad g_{0}=g_{1}
\end{array}\right.
$$


This implies that $p^{*}\left[e_{H}\right]=\left[e_{G}\right]$ up to 2 -torsion, as claimed.

\subsection{Solution of the word problem}

Consider a recursive presentation $\langle S \mid R\rangle$ of some group $G$, where $S$ is a generating set and $R$ the set of relators. A solution to the word problem of the presentation $\langle S \mid R\rangle$ of $G$ consists of an algorithm permitting to decide in a finite amount of steps whether a given word in the alphabet $S$ represents the trivial element of the group $G$, or not. It is known, that, when $G$ has a finite presentation, then all finite presentations of $G$ have simultaneously solvable or nonsolvable word problems and thus one can speak about the word problem of $G$, without making reference to a particular presentation. We slightly anticipate the results in the next sections showing that $T^{\sharp}$ and $T^{*}$ are finitely presented so that the choice of the presentation is irrelevant.

The aim of this section is to give a semantic solution of the word problem. This is not a syntactic solution, that is a solution that uses only algorithms manipulating the letters of the alphabet $S$.

We extend one of the two solutions presented by Artin [1] for the word problem of the braid group. Recall briefly how his solution by representations works: one embeds first the braid group in the automorphism group of a free group and further one decides whether an automorphism of a finitely generated free group is trivial or not, by looking at images of the generators and solving the word problem for words in the free group.

A considerable improvement of Artin's algorithm that uses Dehn's idea (see Penner and Harer [36, 1.2]) of encoding laminations (multi-curves) by tuples of integers was considered by Dynnikov [16] and Malyutin [31]. This turned Artin's solution into a fast and efficient algorithm. We refer to Dynnikov [16, page 1106] and Dehornoy et al [14, Section 8.3] for a thorough discussion of this algorithm and related topics. The methods in $[16 ; 31 ; 14]$ work well in the case of $T^{*}$, as well.

We refer to our paper [21] for a syntactic solution of the word problem derived from the fact that $T^{\sharp}$ is an asynchronously combable group. However, the following one is considerably simpler.

Proposition 2.16 The groups $T^{\sharp}$ and $T^{*}$ have solvable word problem.

Proof The proofs for $T^{\sharp}$ and $T^{*}$ being the same, we only consider the case of $T^{*}$. Consider a word $w$ in the free group generated by the letters $\alpha^{*}, \beta^{*}$, of length $|w|=n$. Our aim is to find an algorithm to decide whether the element represented by $w$ in $T^{*}$ is trivial or not. When this is the case we write $w=T_{T^{*}} 1$. One denotes by $[w]$ the class of the word $w$, as an element of $T^{*}$. 
The main idea is that an element of $T^{*}$ is determined by its action on the fundamental group of a punctured disk whose size is bounded by the size of the element.

Recall that we denoted by $D_{0}^{*}$ the support of $\beta^{*}$ (the central hexagon) and that $D_{n+1}^{*}$ is the result of adding a new hexagon along each boundary component of $D_{n}^{*}$. Thus $D_{n}^{*}$ consists of $3 \cdot 2^{n}-2$ hexagons and thus having $3 \cdot 2^{n+1}-3$ punctures and $3 \cdot 2^{n}$ boundary separating arcs. We will say that the boundary hexagons of $D_{n}^{*}-$ ie those which do not belong to $D_{n-1}^{*}-$ are at distance $n$ from $D_{0}^{*}$. The distance between hexagons is that between the vertices of the dual tree.

Lemma 2.17 If $|w|=n$ then the support of $[w]$ is contained in $D_{n}^{*}$. This means that there exists an admissible subsurface $\Sigma \subset D_{n}^{*}$ such that $[w](\Sigma)$ is also admissible and $[w]: D^{*}-\Sigma \rightarrow D^{*}-[w](\Sigma)$ is rigid. Moreover, $[w](\Sigma) \subset D_{n}^{*}$.

Proof First, we have $\beta^{* m}\left(D_{n}^{*}\right)=D_{n}^{*}$ and $\alpha^{* m}\left(D_{n}^{*}\right) \subset D_{n+1}^{*}$ for any $m$. Recall that $\alpha^{*}$ and $\beta^{*}$ are of finite order.

The claim holds trivially for $n=1$. We use induction on $n$. Thus, if $|w|=n$ and $[w]$ has support $\Sigma$ as claimed, then $\left[\beta^{* m} w\right]$ has support $\Sigma \cup \beta^{*-m}(\Sigma) \subset D_{n}^{*}$. Moreover, $\left[\alpha^{* m} w\right]$ has support $\Sigma \cup \alpha^{*-m}(\Sigma) \subset D_{n+1}^{*}$. The same argument works for $[w](\Sigma)$. This completes the induction step.

Remark 2.18 Actually, we proved that the support of $[w]$ is contained in $D_{k}^{*}$, where $k$ is bounded from above by the number of distinct factors $\alpha^{* m}, \beta^{* m}$ in the word $w$.

First solution, of algebraic nature Consider now the associated word $w(\alpha, \beta)$ obtained from $w$ by replacing $\alpha^{*}, \beta^{*}$ by $\alpha, \beta$, respectively. Then $w(\alpha, \beta)$ is a word representing the image of $[w]$ in the group $T$.

The group $T$ is finitely presented and simple. Therefore, by a well-known result, $T$ has solvable word problem. Thus there exists an algorithm which decides whether $w(\alpha, \beta)={ }_{T} 1$, as we shall assume from now on. Then $[w] \in B_{\infty} \subset T^{\sharp}$ and Lemma 2.17 implies that $[w] \in B\left(D_{n}^{*}\right) \subset B_{\infty}$, where $B\left(D_{n}^{*}\right)$ is the braid group of the $D_{n}^{*}$. Thus it suffices to decide whether the image of $w$ is trivial in $B\left(D_{n}^{*}\right)$, since $[w]$ is rigid on the complement of $D_{n}^{*}$ and thus the identity. Notice however, that $w$ is not given as a word in the generators of the braid group, but as a word in $\alpha^{*}, \beta^{*}$.

Fix a base point $*$ in $D_{n}^{*} \subset D^{*}$ and choose lifts of the mapping classes $\alpha^{*}, \beta^{*}$ which preserve this base point. Then $\alpha^{*}, \beta^{*}$ induce endomorphisms of free groups

$$
\alpha^{*}[2 n]: \pi_{1}\left(D_{2 n}^{*}\right) \rightarrow \pi_{1}\left(D_{2 n}^{*}\right)
$$


by setting $\alpha^{*}[2 n]([\gamma])=1$ if the image of $\gamma$ is not contained within $D_{2 n}^{*}$ (and similarly for $\left.\beta^{*}[2 n]\right)$. Define further $w[2 n](\gamma)=w_{1}[2 n]\left(w_{2}[2 n]\left(\cdots\left(w_{n}[2 n](\gamma)\right) \ldots\right)\right.$. These endomorphisms are well-defined only up to inner automorphisms of the free group. Consider now a system of free generators $\gamma_{1}, \ldots, \gamma_{N}$ for $\pi_{1}\left(D_{n}^{*}\right)$. It follows from Lemma 2.17 that the action of $[w] \in T^{*}$ on the $\gamma_{j}$ is $[w]\left(\gamma_{j}\right)=w[2 n]\left(\gamma_{j}\right)$. In particular, the element $[w]$ is trivial if and only if the outer endomorphism $w[2 n]$ is trivial. Thus we have to check whether there exists $c \in \pi_{1}\left(D_{n}^{*}\right)$ such that

$$
\left(w[2 n]\left(\gamma_{j}\right)\right)_{1 \leq j \leq N}=_{\pi_{1}\left(D_{n}^{*}\right)}\left(c \gamma_{j} c^{-1}\right)_{1 \leq j \leq N} .
$$

This is equivalent to solving the generalized conjugacy problem in the free group $\pi_{1}\left(D_{n}^{*}\right)$. Now, the generalized conjugacy problem is algorithmically solvable for such a group. This holds more generally for biautomatic groups, as it follows immediately from the solution of the usual conjugacy problem (see Word processing in groups [17, Theorem 2.57, page 59] and Gersten and Short [23]). This holds true also for groups satisfying the small cancellation conditions $\mathrm{C}(6), C(4)-T(4), C(3)-T(6)$ (see Bezverkhniǐ [3]).

Second (improved) solution, using laminations The main idea is to use the fact that $T^{*}$ acts as a mapping class group by identifying its action at the level of laminations.

Assume that the punctures are labeled and thus identified with a fixed set $\mathcal{P}$. The mapping classes $\alpha^{*}$ and $\beta^{*}$ induce then (infinite) permutations $\tau_{\alpha}$ and $\tau_{\beta}$ of $\mathcal{P}$.

We consider below the set $\Delta$ consisting of arcs of $D^{*}$ and obtained from the canonical rigid structure by adding copies of each punctured arc on both sides of it, as in the figure below:

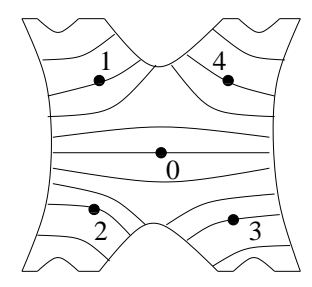

Denote by $\Delta_{n}$ and $\mathcal{P}_{n}$ the restriction of $\Delta$ and $\mathcal{P}$ to the disk $D_{n}^{*}$. In particular we have a natural bijection identification of the set of components of $\Delta_{n}$ and $\mathcal{P}_{n} \times \mathcal{P}_{n} \times \mathcal{P}_{n} \times \mathcal{P}_{n}$.

We consider now a coordinates system encoding the set $\mathcal{C}\left(D_{n}^{*}\right)$ of isotopy classes of simple closed loops on $D_{n}^{*}$ by means of integral vectors. Specifically, for $X \in \mathcal{C}\left(D_{n}^{*}\right)$ one sets

$$
\lambda(X)=(\min |\gamma \cap X|)_{\gamma \in \Delta_{n}} \in \mathbb{Z}^{\Delta_{n}}=\left(\mathbb{Z}^{4}\right)^{\mathcal{P}_{n}}
$$


where || denotes the cardinal, $\gamma$ denotes the arcs of $\Delta_{n}$ and the minimum is taken over all isotopies keeping the extremities fixed and the intersections transverse.

There is an extension to decorated integral laminations [14, Section 8.4] on $D_{n}^{*}$, namely to tuples of disjoint simple closed curves endowed with a labeling, and considered up to isotopy. If $\mathbf{X}$ is a decorated integral lamination consisting of the curves $X_{i}$ with multiplicity $m_{i}$ and set of labels $i \in J$, then

$$
\lambda(\mathbf{X})=\left(m_{i} \lambda\left(X_{i}\right)\right)_{i \in J} \in \bigoplus_{i \in J}\left(\mathbb{Z}^{4}\right)^{\mathcal{P}_{n}} .
$$

It is well-known that $\lambda$ is an embedding and its image is constrained to satisfy the triangle inequalities for the three integers associated to the edges of each hexagon of $\Delta_{n}$ while their sums are even integers.

Let $\mathbf{F}_{n}$ denote the decorated integral lamination consisting of the set of disjoint simple loops $F_{n}(j)$ encircling the punctures labeled $j$, for $j \in \mathcal{P}_{n}$. It is immediate that

$$
\lambda\left(\mathbf{F}_{n}\right)=\bigoplus_{j \in \mathcal{P}_{n}} \mathbf{0} \oplus(1,1,0,0) \oplus \mathbf{0} \in \bigoplus_{j \in \mathcal{P}_{n}}\left(\mathbb{Z}^{4}\right)^{\mathcal{P}_{n}} .
$$

The endomorphism $\boldsymbol{\beta}_{n}^{*}$ Define further two endomorphisms of $\mathbb{Z}^{\Delta_{n}}$, corresponding to the actions of $\alpha^{*}$ and $\beta^{*}$. Recall that $\beta^{*}$ induces a permutation of $\mathcal{P}$ keeping $\mathcal{P}_{n}$ invariant. It makes sense therefore to define

by setting $\quad \boldsymbol{\beta}_{n}^{*}\left(\bigoplus_{j \in \mathcal{P}_{n}} v_{j}\right)=\bigoplus_{j \in \mathcal{P}_{n}} v_{\tau_{\beta}^{-1} j} \in\left(\mathbb{Z}^{4}\right)^{\mathcal{P}_{n}}$

where $v_{j} \in \mathbb{Z}^{4}$ are the 4-dimensional vector components of an element in $\mathbb{Z}^{\Delta_{n}}$ indexed by the same $j \in \mathcal{P}_{n}$.

The endomorphism $\alpha_{n}^{*}$ Further $\alpha^{*}$ also induces a permutation of $\mathcal{P}$ but $\mathcal{P}_{n}$ is not anymore $\tau_{\alpha}$-invariant. Recall that one labeled by 0 the central puncture of the support for $\alpha^{*}$. Define then

$$
\begin{gathered}
\boldsymbol{\alpha}_{n}^{*}: \mathbb{Z}^{\Delta_{n}}=\left(\mathbb{Z}^{4}\right)^{\mathcal{P}_{n}} \rightarrow\left(\mathbb{Z}^{4}\right)^{\mathcal{P}_{n}}=\mathbb{Z}^{\Delta_{n}} \\
\boldsymbol{\alpha}_{n}^{*}\left(\bigoplus_{j \in \mathcal{P}_{n}} v_{j}\right)=\bigoplus_{j \in \mathcal{P}_{n}} w_{j} \in\left(\mathbb{Z}^{4}\right)^{\mathcal{P}_{n}}, \text { with } v_{j}, w_{j} \in \mathbb{Z}^{4}
\end{gathered}
$$$$
\text { by }
$$ 
and $\quad w_{j}= \begin{cases}v_{\tau_{\alpha}^{-1}(j)} & \text { if } j \in \mathcal{P}_{n}-\{0\} \text { and } \tau_{\alpha}^{-1}(j) \in \mathcal{P}_{n} \\ 0 & \text { if } j \in \mathcal{P}_{n}-\{0\} \text { and } \tau_{\alpha}^{-1}(j) \notin \mathcal{P}_{n} \\ \phi\left(v_{0}, v_{1}, v_{2}, v_{3}, v_{4}\right) & \text { if } j=0\end{cases}$

where the function $\phi$ is given below. Each vector component of $\lambda(X)$ is a way to ascribe an integer to every arc of $\Delta_{n}$. The component $v_{0}$ corresponds then to the integers associated to the four arcs near the puncture 0 . Its image $w_{0}$ will depend actually on (some of) the integers associated to the nearby punctures, namely on $v_{1}, v_{2}, v_{3}$ and $v_{4}$. Then we set

$$
\phi\left(v_{0}, v_{1}, v_{2}, v_{3}, v_{4}\right)=\left(E^{\prime}, F^{\prime}, G^{\prime}, H^{\prime}\right) \in \mathbb{Z}^{4}
$$

where $v_{0}=(E, F, G, H), v_{1}=(*, *, *, A), v_{2}=(*, *, *, B), v_{3}=(*, *, *, C)$ and $v_{4}=(*, *, *, D)$ and

$$
\begin{aligned}
& F^{\prime}=\max (E+C, B+F)-G \\
& E^{\prime}=\max (E+D, A+F)-H \\
& G^{\prime}=\max (A+E+C+H, A+B+F+H, B+E+G+H, A+B+F+G) \\
&-(E+G+H) \\
& H^{\prime}=\max (A+C+F+G, C+D+E+G, C+D+E+H, B+D+F+H) \\
&-(F+G+H)
\end{aligned}
$$

A more intuitive definition of $\phi$ is given in the picture below, where we figured out only those labels (of components of $\Delta_{n}$ ) on which the value of $\phi$ actually depends:

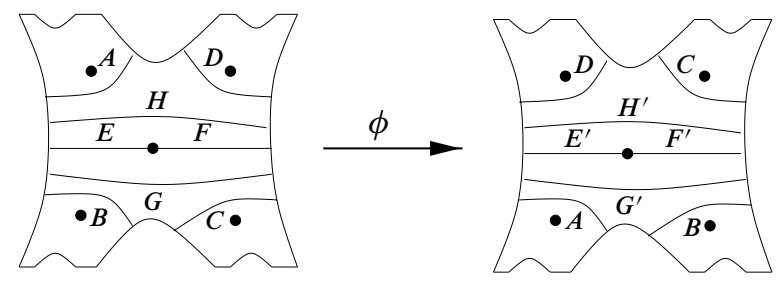

Consider now the word $w=w\left(\alpha^{*}, \beta^{*}\right)$ in the free group with generators $\alpha^{*}$ and $\beta^{*}$. Denote by $w\left(\boldsymbol{\alpha}_{n}^{*}, \boldsymbol{\beta}_{n}^{*}\right)$ the result of substitution of the letters above by the letters $\boldsymbol{\alpha}_{n}^{*}$ and $\boldsymbol{\beta}_{n}^{*}$. Then $w\left(\boldsymbol{\alpha}_{n}^{*}, \boldsymbol{\beta}_{n}^{*}\right)$ is an explicit product of endomorphisms of $\mathbb{Z}^{\Delta_{n}}$.

Proposition 2.19 The word $w=w\left(\alpha^{*}, \beta^{*}\right)$ of length $n$ represents the trivial element in $T^{*}$ if and only if

$$
\lambda\left(\mathbf{F}_{n}\right)=\bigoplus_{j \in \mathcal{P}_{n}} \operatorname{Proj}_{n} \circ w\left(\alpha_{2 n}^{*}, \beta_{2 n}^{*}\right)\left(\lambda\left(F_{n}(j)\right)\right.
$$


where $\operatorname{Proj}_{n}: \mathbb{Z}^{\Delta_{2 n}} \rightarrow \mathbb{Z}^{\Delta_{n}}$ is the projection annihilating all basis elements from $\Delta_{2 n}$ which are not in $\Delta_{n}$.

Proof It suffices to see that the action of $[w]$ on the set $\mathcal{C}\left(D_{n}^{*}\right)$ of isotopy classes of simple closed curves can be described completely in the coordinates system above by the formula on the right hand side.

Lemma 2.20 For each isotopy class $X \in \mathcal{C}\left(D_{n}^{*}\right)$ and $w=w\left(\alpha^{*}, \beta^{*}\right)$ word of length $n$ we have

$$
\lambda([w] X)=\operatorname{Proj}_{n} \circ w\left(\alpha_{2 n}^{*}, \beta_{2 n}^{*}\right)(\lambda(X))
$$

where $[w] X$ denotes the image of $X$ by the mapping class $[w] \in T^{*}$.

Proof Lemma 2.17 shows that the mapping class $[w]$ sends a (class of a) curve in $\mathcal{C}\left(D_{n}^{*}\right)$ into $\mathcal{C}\left(D_{2 n}^{*}\right)$. Therefore one can discard all components outside $\Delta_{2 n}$. The formula above holds for $w=\beta^{*}$ by the very definition of $\boldsymbol{\beta}_{n}^{*}$. The action of $\boldsymbol{\alpha}^{*}$

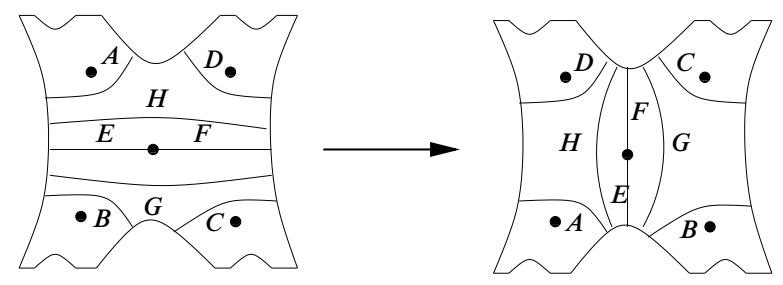

is decomposed into elementary flips:

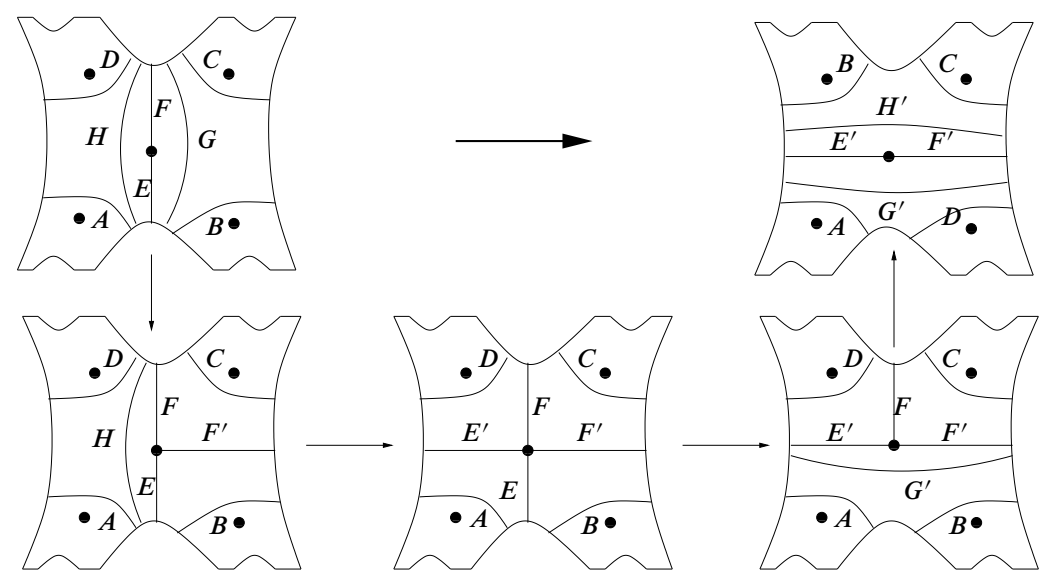


The action of an elementary flip in the coordinates above is well-known $[19 ; 16 ; 36$; $31 ; 14]$ :

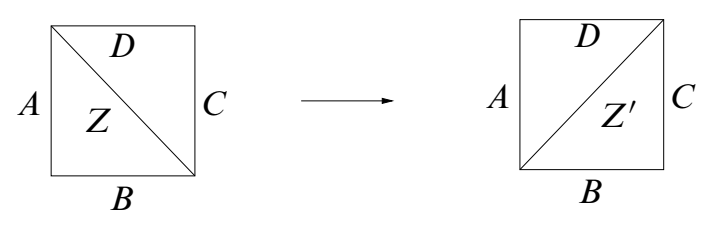

where

$$
Z^{\prime}=\max (A+C, B+D)-Z \text {. }
$$

This ends the proof of the lemma.

Further, assume that the equation in Proposition 2.19 holds. Then the permutation induced by $[w]$ at the level of punctures of $D_{2 n}^{*}$ is trivial. By rigidity of the action outside $D_{2 n}^{*}$ it follows that the image of $w$ in $T$ is the identity element. This is precisely the place where the decoration of the integral lamination is useful. In particular, the element $[w]$ of $T^{*}$ should belong to the subgroup of braids $B\left(D_{n}^{*}\right)$. However it is known that the action of a braid is completely determined by the image of a system of generators for the fundamental group, in particular the image of $\mathbf{F}_{n}$. The equality of coordinates shows that the element $[w]$ acts trivially as automorphism of the fundamental group of $D_{n}^{*}$ and it represents therefore the identity in $T^{*}$.

Remark 2.21 The formulas above express the action of $T^{*}$ on the space of decorated integral laminations and thus on the boundary of the Teichmüller space of $D^{*}$. Notice that this is the tropicalization of the formula expressing the action on the Teichmüller space itself in Penner's lambda coordinates.

Thus Proposition 2.19 furnishes an efficient algorithm for checking whether a given word represents the trivial element of $T^{*}$ or not.

Remark 2.22 The complexity of this algorithm is exponential, because the size of $\mathcal{P}_{n}$ is exponential.

Remark 2.23 The solvability of the word problem is a strong indication that the group has nice properties from algorithmic viewpoint. Notice however that the lamplighter group (the wreath product of $\mathbb{Z} / 2 \mathbb{Z}$ and $\mathbb{Z}$ ) is an infinitely presented 2-generator groups which has solvable word and conjugation problems (see Matthews [33]). Thus the result above does not imply that $T^{*}$ is finitely presented. 


\section{The complex $\mathcal{C}^{+}\left(T^{\sharp}\right)$}

The remainder of the article is devoted to the proof that $T^{\#}$ is finitely presented, by constructing a simply connected cellular complex $\mathcal{C}\left(T^{\sharp}\right)$ on which $T^{\sharp}$ acts cocompactly. Each orbit of 2-cells of this complex will thus correspond to a relation in $T^{\sharp}$. This will enable us to provide an explicit presentation for $T^{\sharp}$. We first introduce an auxiliary complex $\mathcal{C}^{+}\left(T^{\sharp}\right)$, whose simple connectivity is not too difficult to prove, but which is not finite modulo $T^{\sharp}$. The complex $\mathcal{C}\left(T^{\sharp}\right)$ will be a subcomplex of $\mathcal{C}^{+}\left(T^{\sharp}\right)$. We shall finally explain how an analogous construction applies to $T^{*}$.

\subsection{Vertices and edges}

The complex $\mathcal{C}^{+}\left(T^{\sharp}\right)$ is a 2-dimensional cellular complex, whose vertices correspond to the elements of $\mathfrak{R}^{\sharp}$ (see Notation 1.1). The (unoriented) edges correspond to "moves" of two types:

(1) Associativity move, or $A$-move. Let $\mathfrak{r}$ be a rigid structure, and $\gamma$ an arc of $\mathfrak{r}$ which separates two hexagons, say $H_{1}$ and $H_{2}$. Let $\gamma_{1}$ (respectively $\gamma_{2}$ ) be the side of $H_{1}$ (respectively $H_{2}$ ) contained in the boundary of $D^{\sharp}$, and disjoint from $\gamma$. Choose two points $p_{1} \in \gamma_{1}$ and $p_{2} \in \gamma_{2}$. Define the simple arc $\gamma_{0}$, which first connects $p_{1}$ to the puncture of $H_{1}$ (remaining inside $H_{1}$ ), next connects the puncture of $H_{1}$ to the puncture of $H_{2}$, crossing once and transversely the arc $\gamma$, and finally connects the puncture of $\mathrm{H}_{2}$ to $p_{2}$ (remaining inside $\mathrm{H}_{2}$ ). Define an arc by deforming $\gamma_{0}$ around the two punctures, in such a way that it avoids them and separates them. There are exactly two isotopy classes of such arcs, say $\gamma^{\prime}$ and $\gamma^{\prime \prime}$, with free extremities $p_{1}$ and $p_{2}$ (see Figure 7).

One says that the rigid structures $\mathfrak{r}^{\prime}$ and $\mathfrak{r}^{\prime \prime}$, obtained from $\mathfrak{r}$ by only changing $\gamma$ into $\gamma^{\prime}$ or $\gamma^{\prime \prime}$, respectively, are obtained from $\mathfrak{r}$ by an A-move on $\gamma$. A pair as $\left\{\gamma, \gamma^{\prime}\right\}$ or $\left\{\gamma, \gamma^{\prime \prime}\right\}$ determines an edge of type $A$ of $\mathcal{C}^{+}\left(T^{\#}\right)$. Note that there exist exactly two $A$-moves on $\gamma$.

(2) Braiding move, or $B r$-move. Let $\mathfrak{r}$ be a rigid structure, and $\gamma$ an arc of $\mathfrak{r}$ which separates two hexagons, say $H_{1}$ and $H_{2}$. Let $e_{\gamma}$ be a simple arc connecting $q_{1}$ to $q_{2}$ (the punctures of $H_{1}$ and $H_{2}$, respectively), crossing $\gamma$ once and transversely, and contained in $H_{1} \cup H_{2}$. Such an arc is uniquely defined, up to isotopy (fixing $q_{1}$ et $q_{2}$ ). Let $\sigma$ be the positive braid determined by $e_{\gamma}$, which permutes $q_{1}$ and $q_{2}$ (see Definition 2.1). Let $\gamma^{\prime}=\sigma(\gamma)$ be the image of $\gamma$ by $\sigma$, and $\gamma^{\prime \prime}=\sigma^{-1}(\gamma)$ (see Figure 8).

One says that the rigid structures $\mathfrak{r}^{\prime}$ and $\mathfrak{r}^{\prime \prime}$, obtained from $\mathfrak{r}$ by only changing $\gamma$ into $\gamma^{\prime}$ or $\gamma^{\prime \prime}$, respectively, are obtained from $\mathfrak{r}$ by a $B r$-move on $\gamma$. A pair as 


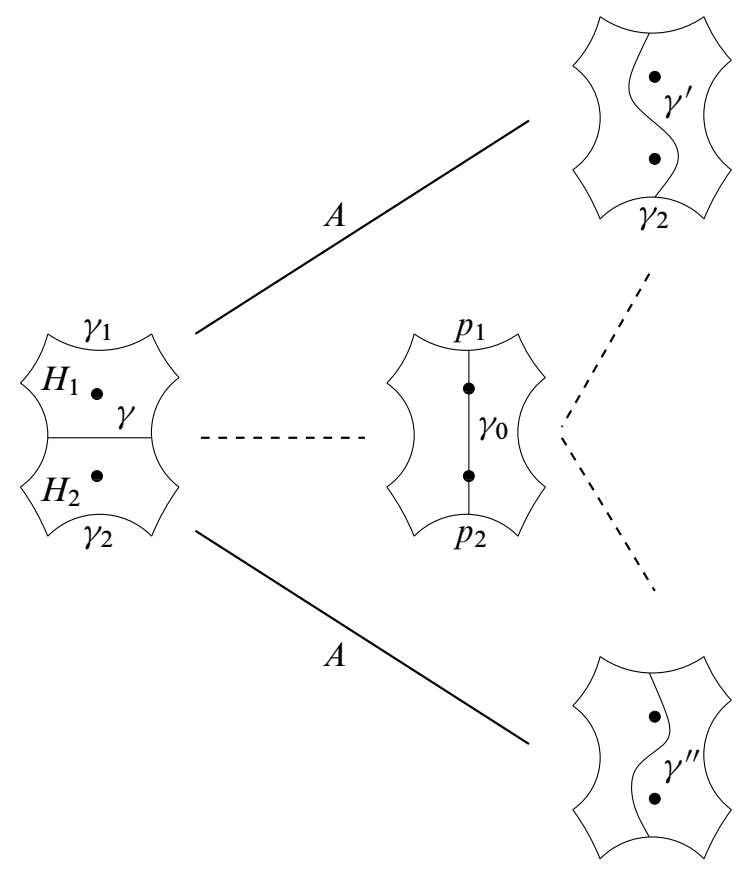

Figure 7: Definition of the $A$-move

$\left\{\gamma, \gamma^{\prime}\right\}$ or $\left\{\gamma, \gamma^{\prime \prime}\right\}$ determines an edge of type $B r$ of $\mathcal{C}^{+}\left(T^{\sharp}\right)$. Note that there exist exactly two $\mathrm{Br}$-moves on $\gamma$.

Definition 3.1 The tree $\mathcal{T}_{\mathfrak{r}}$ of a rigid structure $\mathfrak{r}$ of $D^{\#}$ is the planar tree whose vertices are the punctures of $D^{\sharp}$ and edges are the arcs $e_{\gamma}$ as above, for every arc $\gamma$ of $\mathfrak{r}$.

Note that $\mathcal{T}_{r^{\sharp}}$ is the tree of $D^{\sharp}$.

Remark 3.1 (Orientation of the edges of type $B r$ ) Say that an edge $\gamma \stackrel{B r}{\longrightarrow} \gamma^{\prime}$ is positively oriented if $\gamma^{\prime}$ turns on the left when it approaches the arc $e_{\gamma}$. This means that the braiding $\sigma$ (on the punctures $q_{1}$ and $q_{2}$ ) such that $\gamma^{\prime}=\sigma(\gamma)$, is positive. From now on, a positive braiding will always be denoted by a letter without negative exponent, such as $\sigma, \sigma_{1}$, etc., while $\sigma^{-1}, \sigma_{1}^{-1}$, etc., will refer to negative braidings. On Figure 8, the edge $\gamma \stackrel{B r}{\longrightarrow} \gamma^{\prime}$ is positively oriented, while the edge $\gamma \stackrel{B r}{\longrightarrow} \gamma^{\prime \prime}$ is negatively oriented.

On the contrary, there is no canonical orientation for the edges of type $A$.

From now on we will keep denoting $\alpha$ and $\beta$ for $\alpha^{\sharp}$ and $\beta^{\sharp}$ in the pictures below, when we have to figure out the action of $T^{\sharp}$ on $\mathcal{C}^{+}\left(T^{\sharp}\right)$. 


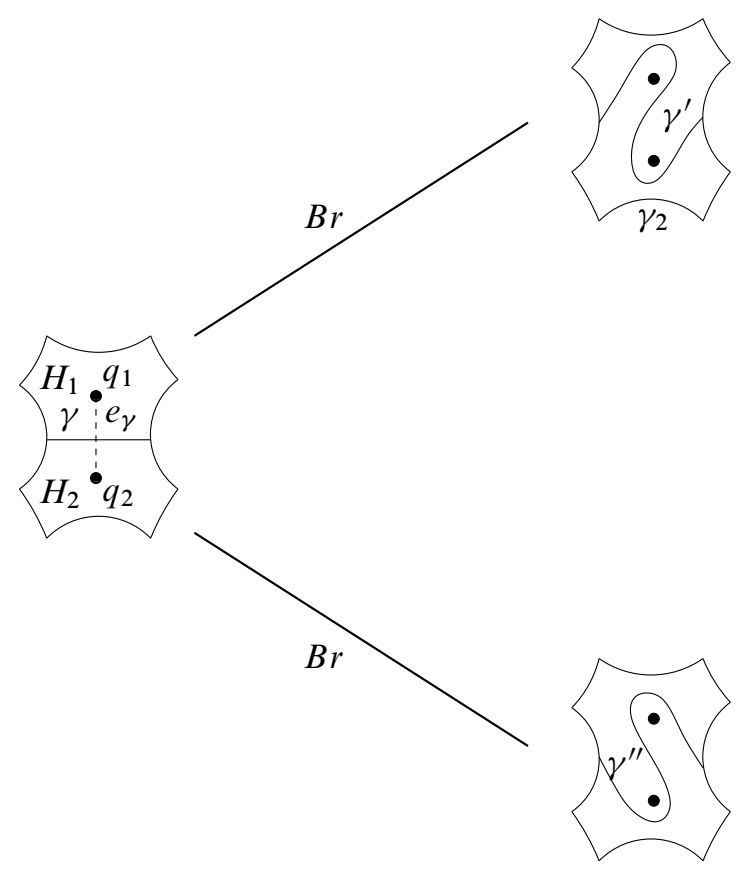

Figure 8: Definition of the $\mathrm{Br}$-move

\subsection{2-cells}

The 2-cells of $\mathcal{C}^{+}\left(T^{\sharp}\right)$ are of the following types:

(1) Cells $A A=B r$. Let $\mathfrak{r}$ be a rigid structure, and $\gamma$ an arc of $\mathfrak{r}$. As we have seen above, there are two edges of type $A$, connecting $\mathfrak{r}$ to $\mathfrak{r}^{\prime}$ and $\mathfrak{r}^{\prime \prime}$. The vertices $\mathfrak{r}^{\prime}$ and $\mathfrak{r}^{\prime \prime}$ are connected by an edge of type $B r$. Thus, one fills in the cycle of three edges $A, A$ and $B r$, by a 2-cell, which is said of type $A A=B r$ (see Figure 9).

(2) Cells of commutation of $A$-moves, $A_{1} A_{2}=A_{2} A_{1}$. Let $\mathfrak{r}$ be a rigid structure, and $H_{1}, H_{2}, H_{1}^{\prime}$ and $H_{2}^{\prime}$ be four distinct hexagons of $\mathfrak{r}$, such that $H_{1}$ and $H_{2}$ (respectively $H_{1}^{\prime}$ and $H_{2}^{\prime}$ ) share a common side $\gamma$ (respectively $\gamma^{\prime}$ ). The commutation of the two $A$-moves, along $\gamma$ and $\gamma^{\prime}$, respectively, generates a square cycle. The point (to be elucidated in Proposition 3.7) is that we only need to fill in the squares of two special types:

- Cells $D C_{1} . H_{2}$ and $H_{1}^{\prime}$ share a common side, see Figure 10.

- Cells $D C_{2}$. $H_{2}$ and $H_{1}^{\prime}$ are separated by a hexagon $H_{0}$; see Figure 11. 


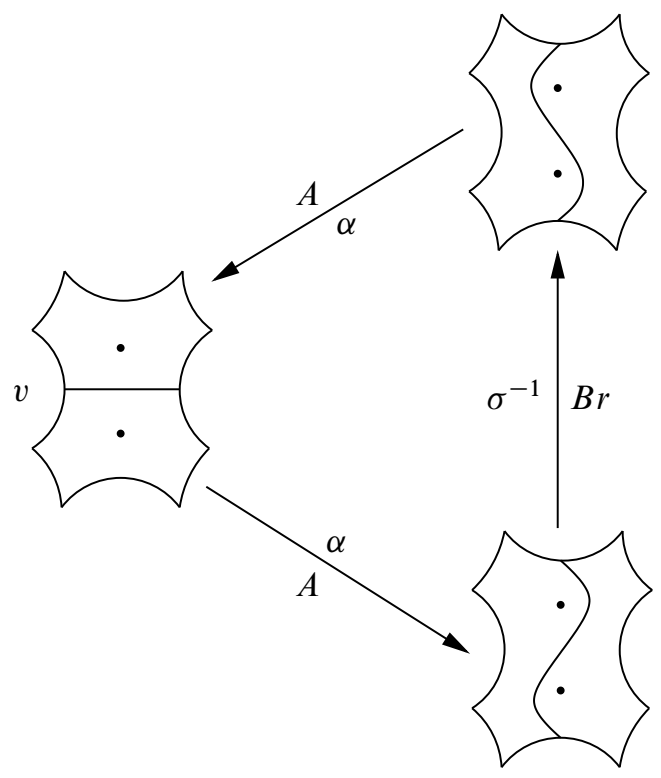

Figure 9: Cycle $A A=B r$

(3) Pentagonal cells. Let $\mathfrak{r}$ be a rigid structure, and $H_{1}, H_{2}$ and $H_{3}$ be three hexagons of $\mathfrak{r}$, such that $H_{1}$ and $H_{2}$ are adjacent along a side $\gamma$, and $H_{3}$ and $H_{2}$ are adjacent along a side $\delta$. There is a cycle of five $A$-moves, which only involves the $\operatorname{arcs} \gamma$ and $\delta$ of $\mathfrak{r}$; see Figure 12. It is filled in, producing a 2-cell of pentagonal type.

(4) Cells coming from the presentation of the braid group. Recall that there is a general theorem of V Sergiescu [38] which can be used to provide a presentation for $B_{\infty}$ with generators the positive braidings along the edges of the tree of $D^{\sharp}$ or the tree $\mathcal{T}_{\mathfrak{r}}$ of any rigid structure $\mathfrak{r}$. Let $\mathfrak{r}$ be a rigid structure, and $\mathcal{T}_{\mathfrak{r}}$ be its tree.

(a) Hexagonal cells. Let $e_{1}$ and $e_{2}$ be two edges of $\mathcal{T}_{\mathfrak{r}}$, which are incident to a puncture $p$. Let $\sigma_{1}$ and $\sigma_{2}$ be the braidings along $e_{1}$ and $e_{2}$, respectively. Then $\sigma_{1} \sigma_{2} \sigma_{1}=\sigma_{2} \sigma_{1} \sigma_{2}$. Let $B r_{1}$ and $B r_{2}$ denote the braiding moves, corresponding to $\sigma_{1}$ and $\sigma_{2}$, respectively. In terms of $B r$-moves, the braid relation becomes

$$
B r_{2} B r_{1} B r_{2}=B r_{1} B r_{2} B r_{1} \text {, }
$$

and one adds a $2-$ cell to fill in the cycle of those 6 braiding moves.

(b) Octagonal cells. Let $e_{1}, e_{2}$ and $e_{3}$ be the three edges which are incident to a puncture $p$. Suppose that their enumeration respects the cyclic counterclockwise 


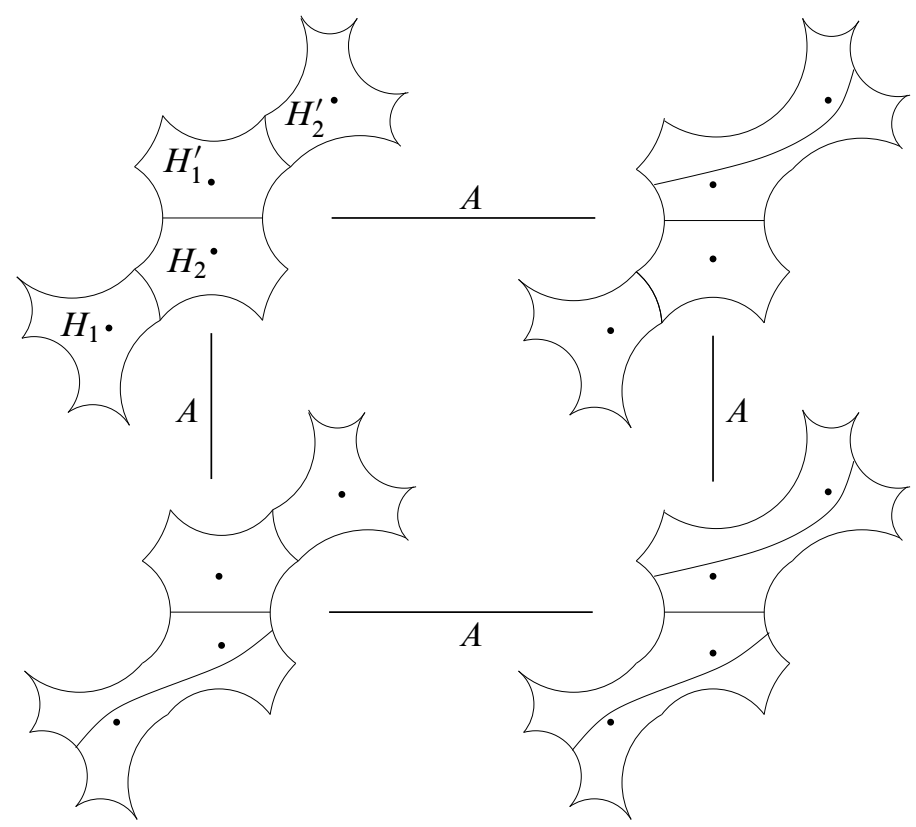

Figure 10: Cycle of type $D C_{1}$

orientation of the planar surface around $p$. Using notation as in a), one has the relation $\sigma_{1} \sigma_{2} \sigma_{3} \sigma_{1}=\sigma_{2} \sigma_{3} \sigma_{1} \sigma_{2}=\sigma_{3} \sigma_{1} \sigma_{2} \sigma_{3}$. In terms of $B r$-moves, this gives

$$
B r_{3} B r_{2} B r_{1} B r_{3}=B r_{2} B r_{1} B r_{3} B r_{2}=B r_{1} B r_{3} B r_{2} B r_{1} \text {, }
$$

and one adds 2-cells to fill in the corresponding cycles of 8 braidings.

(c) Squares. Let $e_{1}$ and $e_{2}$ two disjoint edges. Then $\sigma_{1} \sigma_{2}=\sigma_{2} \sigma_{1}$. In terms of braiding moves, this gives

$$
B r_{1} B r_{2}=B r_{2} B r_{1},
$$

and one adds a 2 -cell to fill in this square cycle.

(5) Cells of commutation of $A$-moves with $B r$-moves. Let $\mathfrak{r}$ be a rigid structure. An $A$-move along an arc $\gamma$ commutes with a $B r$-move along an edge $e$ of $\mathcal{T}_{\mathfrak{r}}$ if $\gamma$ and $e$ are disjoint. Thus, there is a square cycle of the form

$$
A B r=B r A
$$

which one fills in by a $2-$ cell.

We note that the minimum level (see Definition 3.2) for such a cell is 5; see Figure 16. 


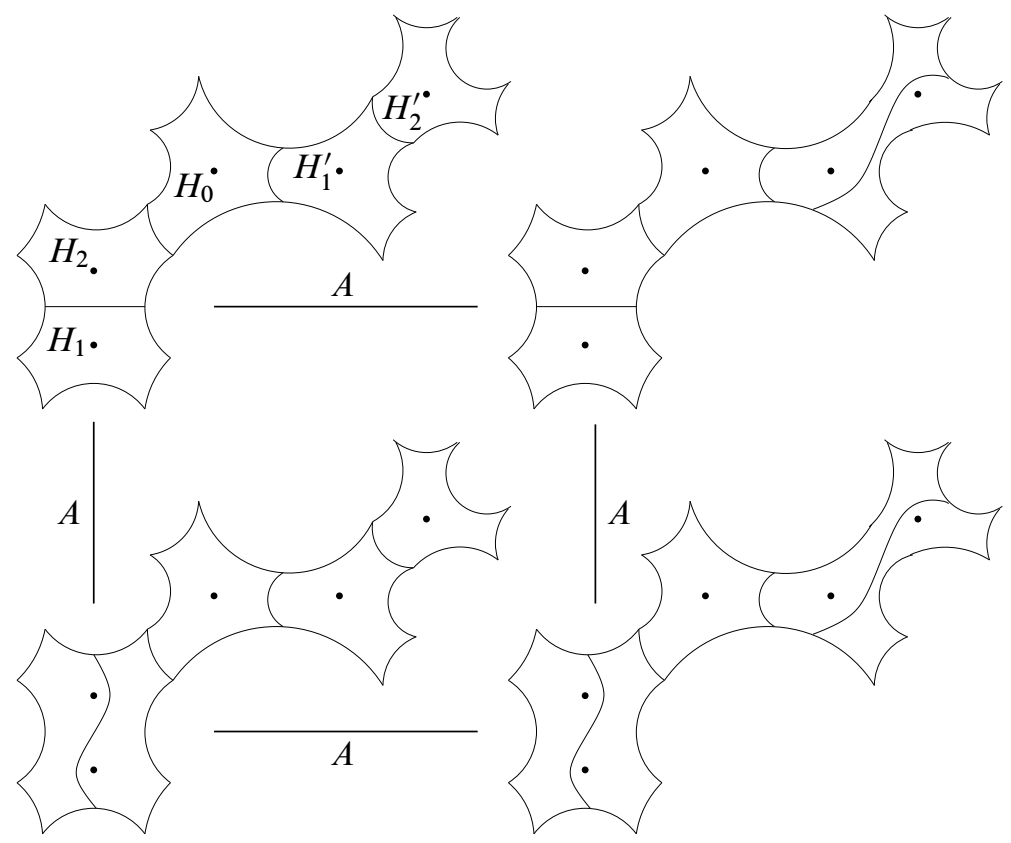

Figure 11: Cycle of type $D C_{2}$

Definition 3.2 Let $\omega$ be a 2-cell, and $r$ a vertex of the boundary cycle $\partial \omega$. The vertices of $\partial \omega$ differ from $r$ by a finite number of $\operatorname{arcs} \gamma$. The support of $\omega$ is the minimal connected subsurface of $D^{\sharp}$ which is a union of hexagons of $r$ and contains all the arcs $\gamma$. The level of the 2-cell $\omega$ is the number of arcs of $r$ which belong to the boundary of the support of $\omega$.

The description of $\mathcal{C}^{+}\left(T^{\sharp}\right)$ is now complete, and the following is obvious:

Proposition 3.2 The complex $\mathcal{C}^{+}\left(T^{\sharp}\right)$ is a $T^{\sharp}$-complex.

\subsection{Connectivity of $\mathcal{C}^{+}\left(T^{\sharp}\right)$}

We first recall a useful lemma of algebraic topology [2, Proposition 6.2]; see also a variant of it in [20], which we have used already in [22].

Lemma 3.3 Let $\mathcal{M}$ and $\mathcal{C}$ be two $C W$-complexes of dimension 2, with oriented edges, and $f: \mathcal{M}^{(1)} \rightarrow \mathcal{C}^{(1)}$ be a cellular map between their 1-skeletons, which is surjective on 0 -cells and 1-cells. Suppose that: 


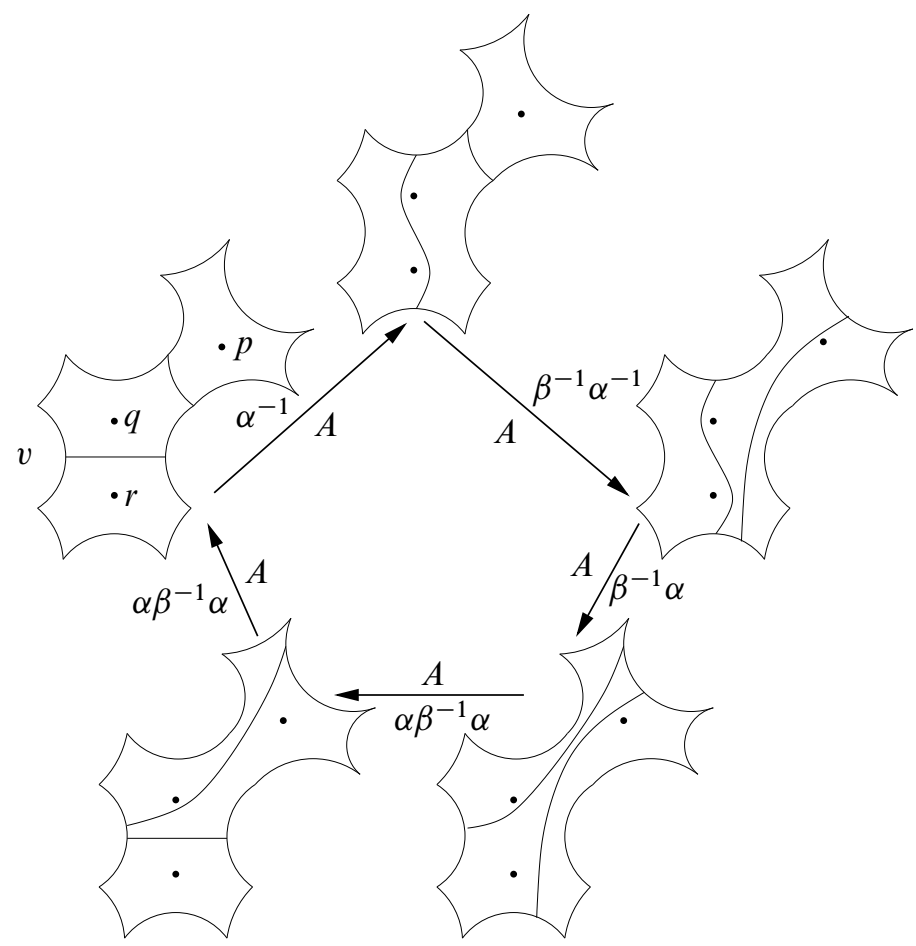

Figure 12: Pentagonal cycle

(1) $\mathcal{C}$ is connected and simply connected;

(2) For each vertex $c$ of $\mathcal{C}, f^{-1}(c)$ is connected and simply connected;

(3) Let $c_{1} \stackrel{e}{\longrightarrow} c_{2}$ be an oriented edge of $\mathcal{C}$, and let $m_{1}^{\prime} \stackrel{e^{\prime}}{\longrightarrow} m_{2}^{\prime}$ and $m_{1}^{\prime \prime} \stackrel{e^{\prime \prime}}{\longrightarrow} m_{2}^{\prime \prime}$ be two lifts in $\mathcal{M}$. Then we can find two paths $m_{1}^{\prime} \stackrel{p_{1}}{\longrightarrow} m_{1}^{\prime \prime}$ in $f^{-1}\left(c_{1}\right)$ and $m_{2}^{\prime} \stackrel{p_{2}}{\longrightarrow} m_{2}^{\prime \prime}$ in $f^{-1}\left(c_{2}\right)$ such that the loop

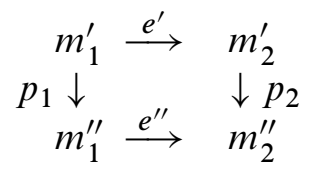

is contractible in $\mathcal{M}$;

(4) For any 2-cell $X$ of $\mathcal{C}$, its boundary $\partial X$ can be lifted to a contractible loop of $\mathcal{M}$.

Then $\mathcal{M}$ is connected and simply connected. 


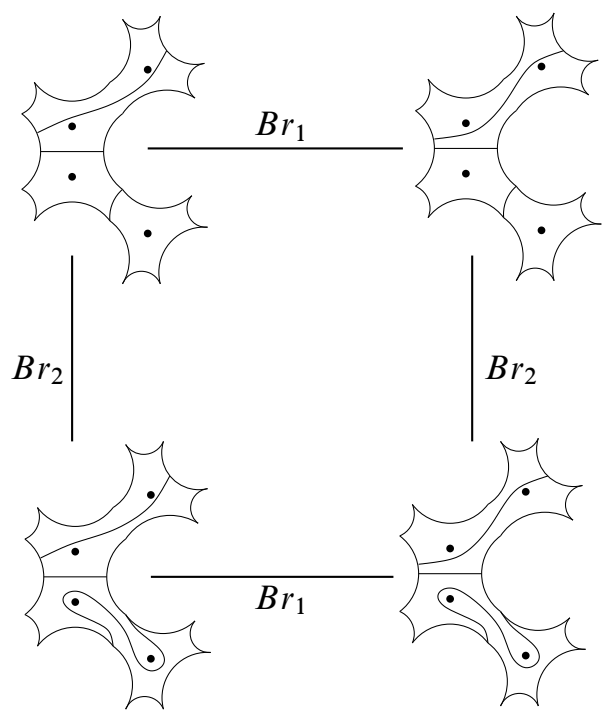

Figure 13: Commutation $B r_{1} B r_{2}=B r_{2} B r_{1}$ of level 6

Recall that $D$ is the surface $D^{\sharp}$ viewed without its punctures. We will use Lemma 3.3 to study a certain cellular map $f: \mathcal{C}^{+}\left(T^{\sharp}\right) \rightarrow \mathcal{H} \mathcal{T}_{\text {red }}(D)$.

Definition 3.3 The reduced Hatcher-Thurston complex $\mathcal{H} \mathcal{T}_{\text {red }}(D)$ is a 2-dimensional cellular complex whose vertices are the rigid structures of $D$, whose edges correspond to $A$-moves, and whose 2-cells are of three types: $D C_{1}, D C_{2}$, and pentagonal cells. The definition of the $A$-move in $\mathcal{H} \mathcal{T}_{\text {red }}(D)$ is deduced from the definition of the $A$-move in $\mathcal{C}^{+}\left(T^{\sharp}\right)$ by forgetting the punctures.

Note that, if $\gamma$ is an arc of a rigid structure of $D$, there is a unique $A$-move on $\gamma$.

Remark 3.4 (1) If $\Sigma_{0, \infty}$ is the surface without boundary obtained by gluing along their boundaries two copies of $D$ with opposite orientations, then $\mathcal{H} \mathcal{T}_{\text {red }}(D)$ is a subcomplex of the reduced Hatcher-Thurston complex $\mathcal{H} \mathcal{T}_{\text {red }}\left(\Sigma_{0, \infty}\right)$ of the surface $\Sigma_{0, \infty}$, as it appears in [22, Definition 5.2]. The argument used in [22] to prove that $\mathcal{H} \mathcal{T}_{\text {red }}\left(\Sigma_{0, \infty}\right)$ is connected and simply connected actually reduces to proving that $\mathcal{H} \mathcal{T}_{\text {red }}(D)$ is connected and simply connected. The point of that proof (see Proposition 5.5 in [22]) is that there is a surjection of the Cayley complex of Thompson's group $T$, for the presentation with generators $\alpha$ and $\beta$, onto the complex $\mathcal{H} \mathcal{T}_{\text {red }}(D)$. This is essentially used to show that the square cycles generated by the commutations of any two $A$-moves are filled in by $2-$ cells of types $D C_{1}, D C_{2}$, and by pentagons. 


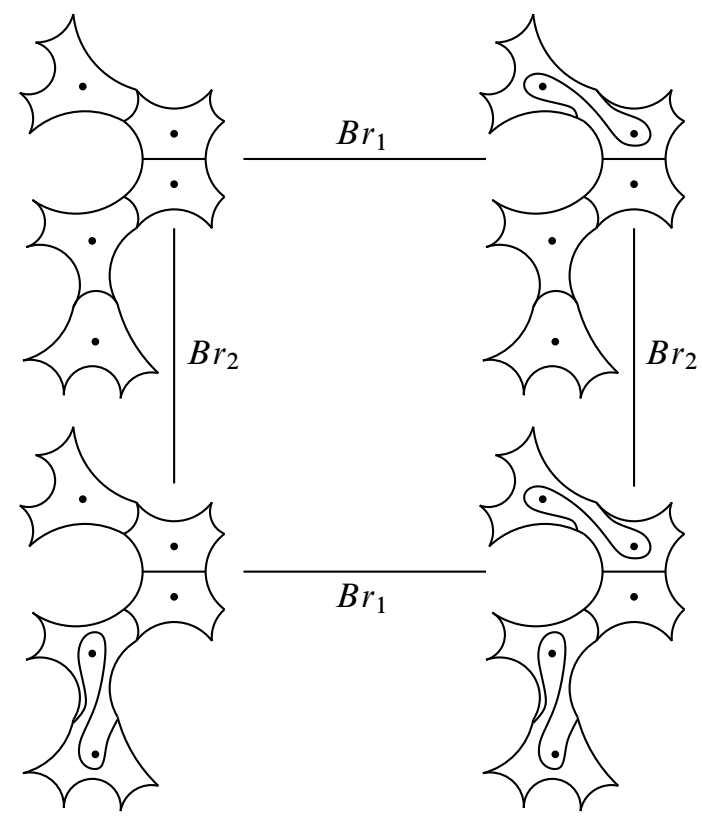

Figure 14: First commutation $B r_{1} B r_{2}=B r_{2} B r_{1}$ of level 7

(2) The complex $\mathcal{H} \mathcal{T}_{\text {red }}(D)$ is a $T$-complex, and $\mathcal{H} \mathcal{T}_{\text {red }}(D) / T$ has one vertex, one edge, and three 2-cells: the two squares $D C_{1}$ and $D C_{2}$, and the pentagon.

The following is obvious:

Proposition 3.5 There is a well defined cellular map

$$
f: \mathcal{C}^{+}\left(T^{\sharp}\right) \rightarrow \mathcal{H} \mathcal{T}_{\text {red }}(D),
$$

which is induced by forgetting the punctures. The map $f$ is $\left(T^{\sharp}, T\right)$-equivariant.

Definition 3.4 The $T^{\sharp}$-type of a 2-cell $\omega$ of $\mathcal{C}^{+}\left(T^{\sharp}\right)$ is its image in $\mathcal{C}^{+}\left(T^{\sharp}\right) / T^{\sharp}$. The $T$-type of $\omega$ is $f(\omega) \bmod T$ in $\mathcal{H} \mathcal{T}_{\text {red }}(D) / T$.

Proposition 3.6 There is exactly one $T$-type of 2-cells $D C_{1}$, one $T$-type of 2-cells $D C_{2}$, and one $T$-type of pentagonal 2-cells. Each $T$-type of 2-cell corresponds to finitely many different $T^{\sharp}$-types. In other words, if $\omega$ is a $2-$ cell in $\mathcal{H} \mathcal{T}_{\text {red }}(D)$, then the set of 2-cells in $\mathcal{C}^{+}\left(T^{\sharp}\right)$ which are the preimages of $\omega$ by $f$ is finite modulo $T^{\sharp}$. 


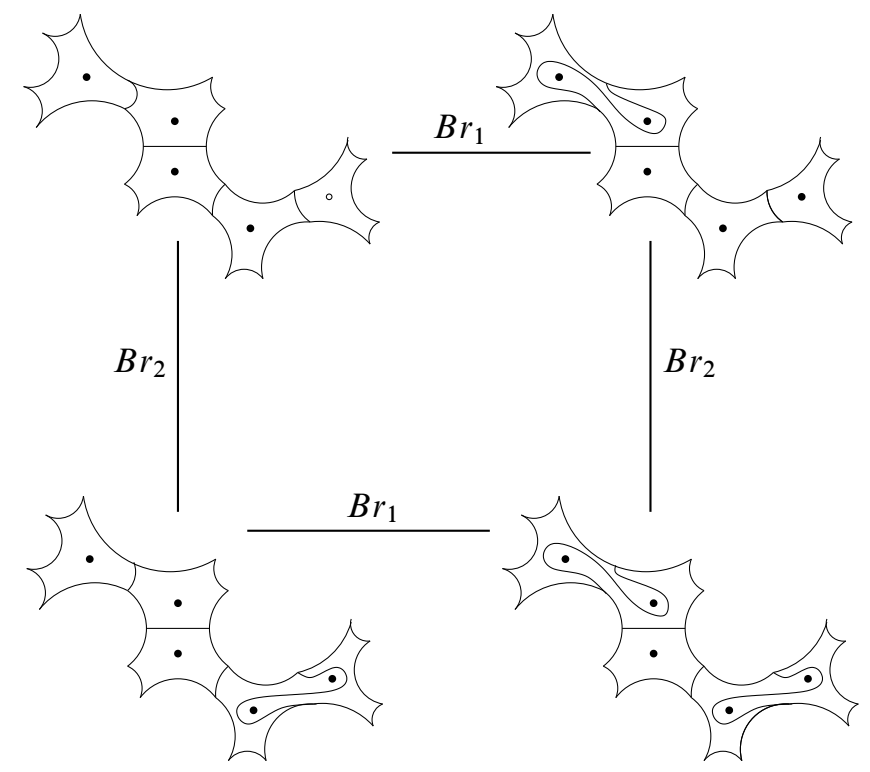

Figure 15: Second commutation $B r_{1} B r_{2}=B r_{2} B r_{1}$ of level 7

Proof The first assertion was already mentioned in Remark 3.4 (2). The second is related to the fact that an edge of type $A$ in $\mathcal{H T}_{\text {red }}(D)$ admits two lifts in $\mathcal{C}^{+}\left(T^{\sharp}\right)$ with the same origin (see Figure 9). Therefore, a $2-$ cell in $\mathcal{H} \mathcal{T}_{\text {red }}(D)$ bounded by a cycle of $n$ edges admits at most $2^{n-1}$ lifts in $\mathcal{C}^{+}\left(T^{\sharp}\right)$ based at the same origin.

Proposition 3.7 The complex $\mathcal{C}^{+}\left(T^{\sharp}\right)$ is connected and simply connected.

Proof Let us apply Lemma 3.3 to the map $f$. Condition (1) is fulfilled. The preimage by $f$ of a vertex $\mathfrak{r}$ of $\mathcal{H} \mathcal{T}_{\text {red }}\left(D^{\sharp}\right)$ is isomorphic to the Cayley complex of the group $B_{\infty}$, for the presentation of Sergiescu associated to the tree $\mathcal{T}_{\mathfrak{r}}$ of $\mathfrak{r}$. Consequently, it is connected and simply connected, and condition (2) of Lemma 3.3 is fulfilled.

Let us examine condition (3). The edges $e^{\prime}$ and $e^{\prime \prime}$ are of type $A$. Since $m_{1}^{\prime}$ (respectively $m_{2}^{\prime}$ ) is connected to $m_{1}^{\prime \prime}$ (respectively $m_{2}^{\prime \prime}$ ) by a sequence of edges of type $B r$, it suffices to consider the case when $p_{1}$ is an edge of type $B r$. But this forces $p_{2}$ either to be trivial (the loop $p_{1} e^{\prime \prime} e^{-1}$ bounds a 2-cell $A A=B r$ ) or to be an edge of type $\mathrm{Br}$ (the loop $e^{\prime} p_{2} e^{\prime \prime-1} p_{1}^{-1}$ bounds a 2-cell $A B r=B r A$ ).

Condition (4) is obviously fulfilled, by definition of $\mathcal{C}^{+}\left(T^{\sharp}\right)$. To conclude, $\mathcal{C}^{+}\left(T^{\sharp}\right)$ is connected and simply connected. 


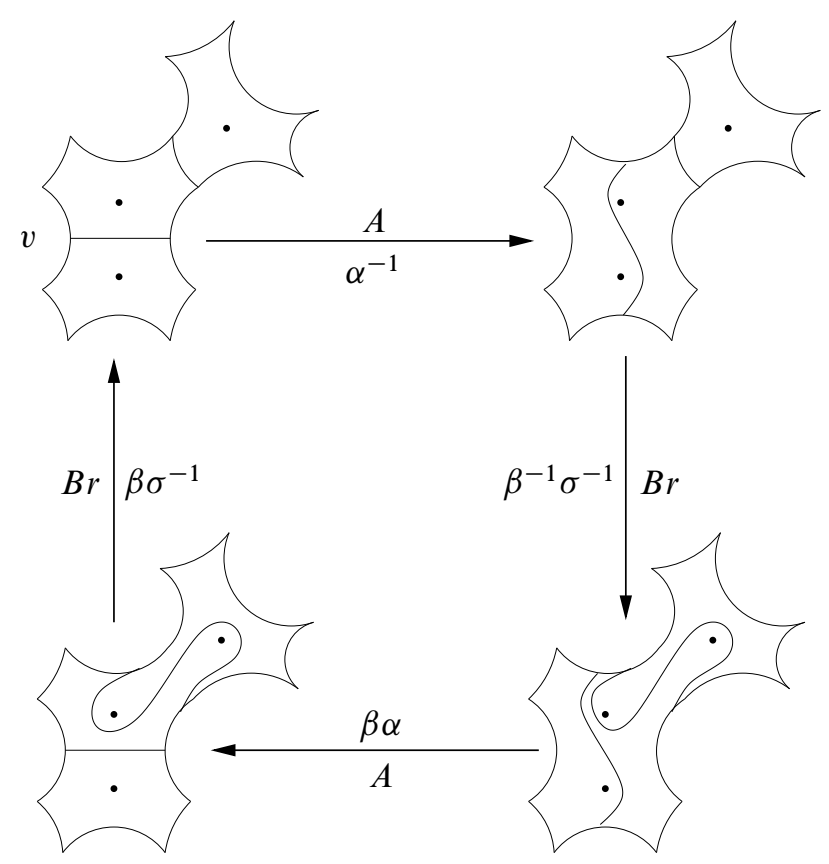

Figure 16: Cycle $B r A=A B r$ of level 5

\section{The reduced complex $\mathcal{C}\left(T^{\sharp}\right)$ and a presentation for $T^{\sharp}$}

\subsection{Simple connectivity of $\mathcal{C}\left(T^{\sharp}\right)$}

Definition 4.1 The reduced complex $\mathcal{C}\left(T^{\sharp}\right)$ is the subcomplex of $\mathcal{C}^{+}\left(T^{\sharp}\right)$ which has the same 1-skeleton as $\mathcal{C}^{+}\left(T^{\sharp}\right)$. The 2-cells are of the following types:

(1) $A A=B r$ (Figure 9), $D C_{1}$ of Figure 10, $D C_{2}$ of Figure 11, pentagon of Figure 12;

(2) $A B r=B r A$ of level 5 of Figure 16, level 6 of Figure 18, and level 7 of Figures 21 and 22;

(3) $B r_{1} B r_{2}=B r_{2} B r_{1}$ coming from the commutation of certain braidings with disjoint supports: cells of level 6 of the $T^{\sharp}$-type of Figure 13, and cells of level 7 the $T^{\sharp}$-type of Figure 14 and of the $T^{\sharp}$-type of Figure 15);

(4) Cells coming from the braid group: $B r_{1} B r_{2} B r_{2}=B r_{2} B r_{1} B r_{2}$ (Section 3.2 (4)(a)) and $B r_{3} B r_{2} B r_{1} B r_{3}=B r_{2} B r_{1} B r_{3} B r_{2}=B r_{1} B r_{3} B r_{2} B r_{1}$ (Section 3.2 (4)(b)). 
The point is that $\mathcal{C}\left(T^{\sharp}\right)$, contrary to $\mathcal{C}^{+}\left(T^{\sharp}\right)$, contains finitely many $T^{\sharp}$-types of cells $A B r=B r A$ and $B r_{1} B r_{2}=B r_{2} B r_{1}$. It follows that the quotient $\mathcal{C}\left(T^{\sharp}\right) / T^{\sharp}$ is finite. Moreover:

Proposition 4.1 The complex $\mathcal{C}\left(T^{\sharp}\right)$ is connected and simply connected.

Proof Since $\mathcal{C}^{+}\left(T^{\sharp}\right)$ is connected and has the same 1 -skeleton as $\mathcal{C}\left(T^{\sharp}\right)$, the latter is connected as well. To prove the simple connectivity of $\mathcal{C}\left(T^{\sharp}\right)$ from the simple connectivity of $\mathcal{C}^{+}\left(T^{\sharp}\right)$, it suffices to check that the cycles bounding the 2-cells which belong to $\mathcal{C}^{+}\left(T^{\sharp}\right)$ but not to $\mathcal{C}\left(T^{\sharp}\right)$ may be filled in by some combinations of 2-cells of $\mathcal{C}\left(T^{\sharp}\right)$ only.

Note first that for each of the three $T$-types ( $D C_{1}, D C_{2}$ or pentagon), we have selected a unique $T^{\sharp}$-type of lift in $\mathcal{C}\left(T^{\sharp}\right)$ (compare with Proposition 3.6). However:

Lemma 4.2 Let $\omega$ be any 2 -cell of $\mathcal{C}^{+}\left(T^{\sharp}\right)$ of $T$-type $D C_{1}, D C_{2}$, or of pentagonal $T$-type. Then $\partial \omega$ is filled in by $2-$ cells which belong to $\mathcal{C}\left(T^{\sharp}\right)$, hence is homotopically trivial in $\mathcal{C}\left(T^{\sharp}\right)$.

Proof Let us introduce the following terminology. Suppose that the boundary of a $2-$ cell $\omega^{\prime}$ is filled in by some $2-$ cells $\omega, \omega_{1}, \ldots, \omega_{n}$. Then we will say that $\omega^{\prime}$ is equivalent to $\omega$ modulo $\omega_{1}, \ldots, \omega_{n}$.

Let us consider the $T^{\sharp}$-types of cells of $T$-type $D C_{1}$. The only $T^{\sharp}$-type which belongs to $\mathcal{C}\left(T^{\sharp}\right)$ is that of Figure 10. Yet, one would obtain another $T^{\sharp}$-type by changing the lift of the horizontal or of the vertical edge (based at the same top left corner of the square). The symmetry of the square makes it sufficient to restrict to the horizontal edge case. Thus, another $T^{\sharp}$-type is represented in Figure 17: it is the large cell which is filled in by one cell $D C_{1}$ (of Figure 10), two cells of type $A A=B r$, and one cell $A B r=B r A$ of level 6 (the small square of Figure 18). The point is that all of them belong to $\mathcal{C}\left(T^{\sharp}\right)$, so that the boundary of the large cell is homotopically trivial in $\mathcal{C}\left(T^{\sharp}\right)$.

Note that another $T^{\sharp}$-type would be obtained by changing the vertical edge of the large cell of Figure 17. But we would prove, by the same puzzle game as above, that its boundary is homotopically trivial in $\mathcal{C}\left(T^{\sharp}\right)$. However, there is a subtlety here: the piece $A B r=B r A$ of level 6 we would use is not $T^{\#}$-equivalent to that used above (ie the small square of Figure 18). This inequivalent piece $A B r=B r A$ is the large square that is represented in Figure 18. But the same figure shows that the latter is equivalent to the cell $A B r=B r A$ (belonging to $\mathcal{C}\left(T^{\sharp}\right)$ ), modulo some cells which all belong to $\mathcal{C}\left(T^{\sharp}\right)$. Indeed, the large square is filled in by the small piece $A B r=B r A$, 


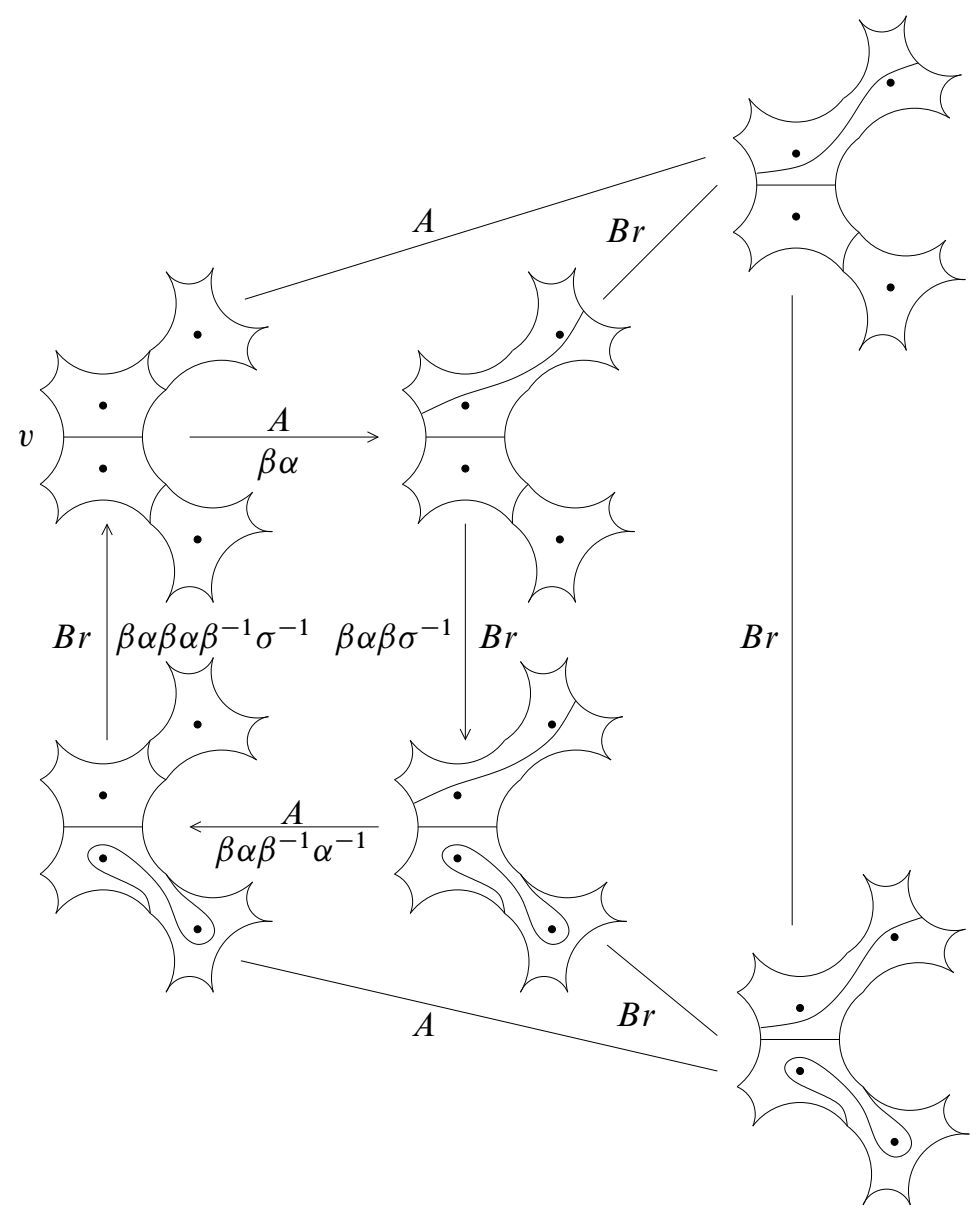

Figure 17: Relation between two inequivalent cycles of type $D C_{1}$

two cells $A A=B r$ and one cell $B r_{1} B r_{2}=B r_{2} B r_{1}$ of level 6 . All of them do belong to $\mathcal{C}\left(T^{\sharp}\right)$.

Let us now consider the $T^{\sharp}$-types of cells of $T$-type $D C_{2}$. The only $T^{\sharp}$-type which belongs to $\mathcal{C}\left(T^{\sharp}\right)$ is that of Figure 11. One would obtain different $T^{\sharp}$-types by changing the lift of the horizontal edge (see the large cell of Figure 19) or of the vertical edge (see the large cell of Figure 20). Using cells of type $D C_{2}$ of Figure 11, of type $A A=B r$ and of type $A B r=B r A$ of level 7 (of Figure 21 and Figure 22) as puzzle pieces (which all belong to $\mathcal{C}\left(T^{\sharp}\right)$ ), one proves that the large cells of Figure 19 and Figure 20 are equivalent to the small ones modulo cells which are in $\mathcal{C}\left(T^{\sharp}\right)$. 


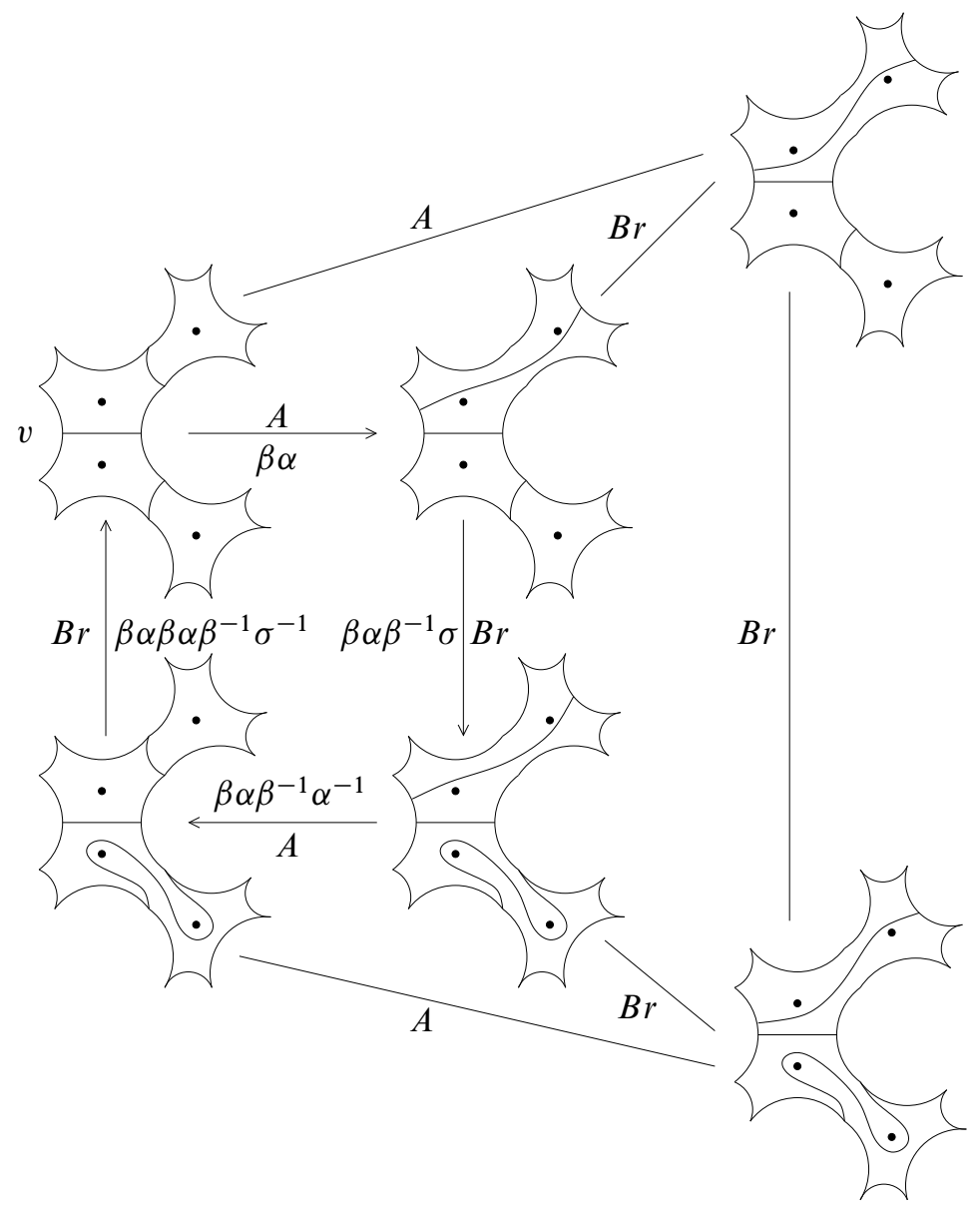

Figure 18: Relation between two inequivalent cycles $A B r=B r A$ of level 6

Note that one could obtain other $T^{\sharp}$-types of cells of $T$-type $D C_{2}$ by changing both the horizontal and the vertical edges. The pieces $A B r=B r A$ of level 7 we would need might not be $T^{\sharp}$-equivalent to those of Figure 21 and Figure 22, but equivalent to the latter modulo cells $A A=B r$ and $B r_{1} B r_{2}=B r_{2} B r_{1}$ of level 7 (Figure 14 and Figure 15).

Let us finally consider the $T^{\sharp}$-types of cells of pentagonal $T$-type. The only $T^{\sharp}$-type which belongs to $\mathcal{C}\left(T^{\sharp}\right)$ is that of Figure 12. The other ones (see the large pentagonal cell of Figure 23) are equivalent to it modulo cells of type $A A=B r$ and of type $A B r=B r A$ of level 5 (of Figure 16), which all belong to $\mathcal{C}\left(T^{\sharp}\right)$. 


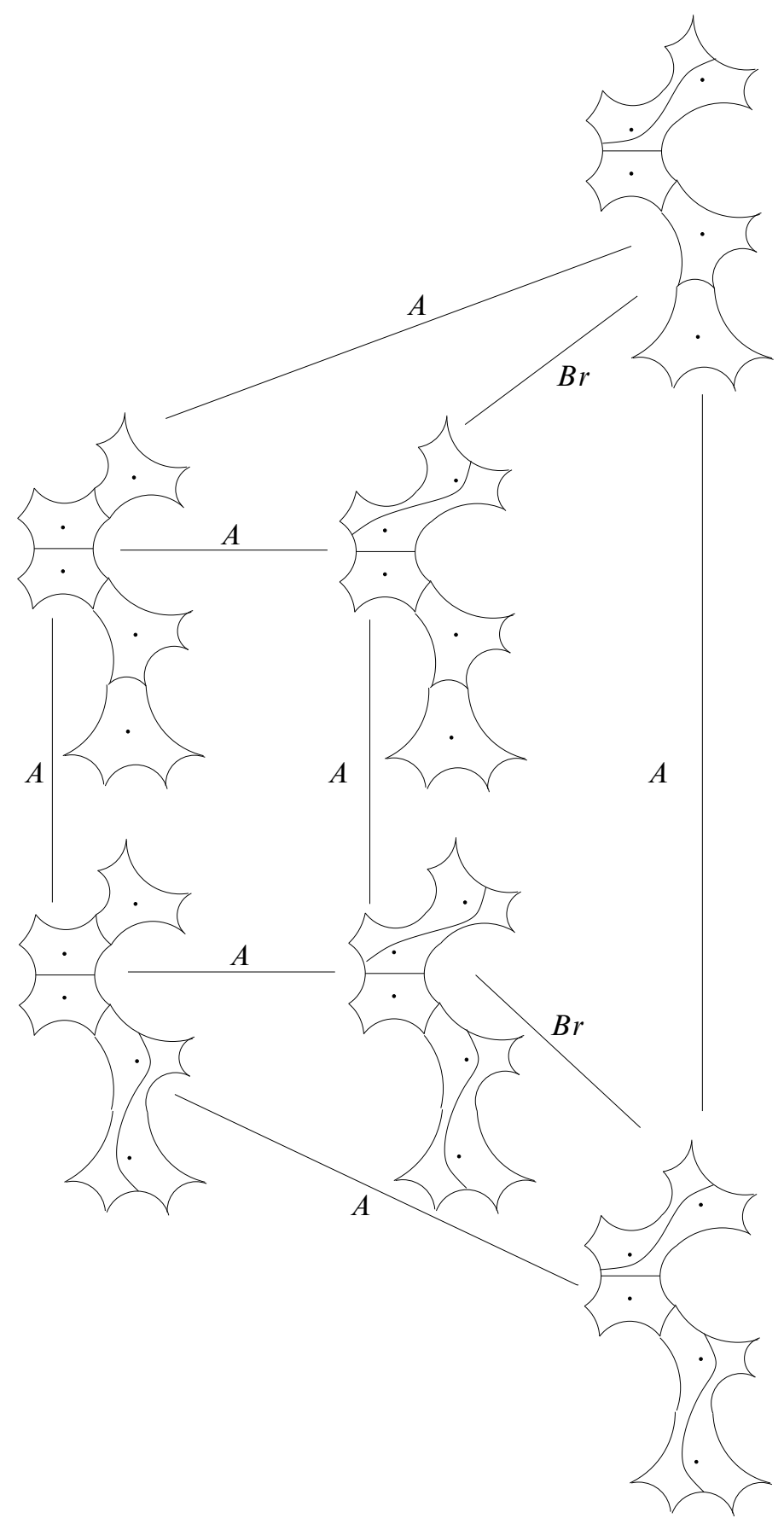

Figure 19: Relation between two inequivalent cycles of type $D C_{2}$ 


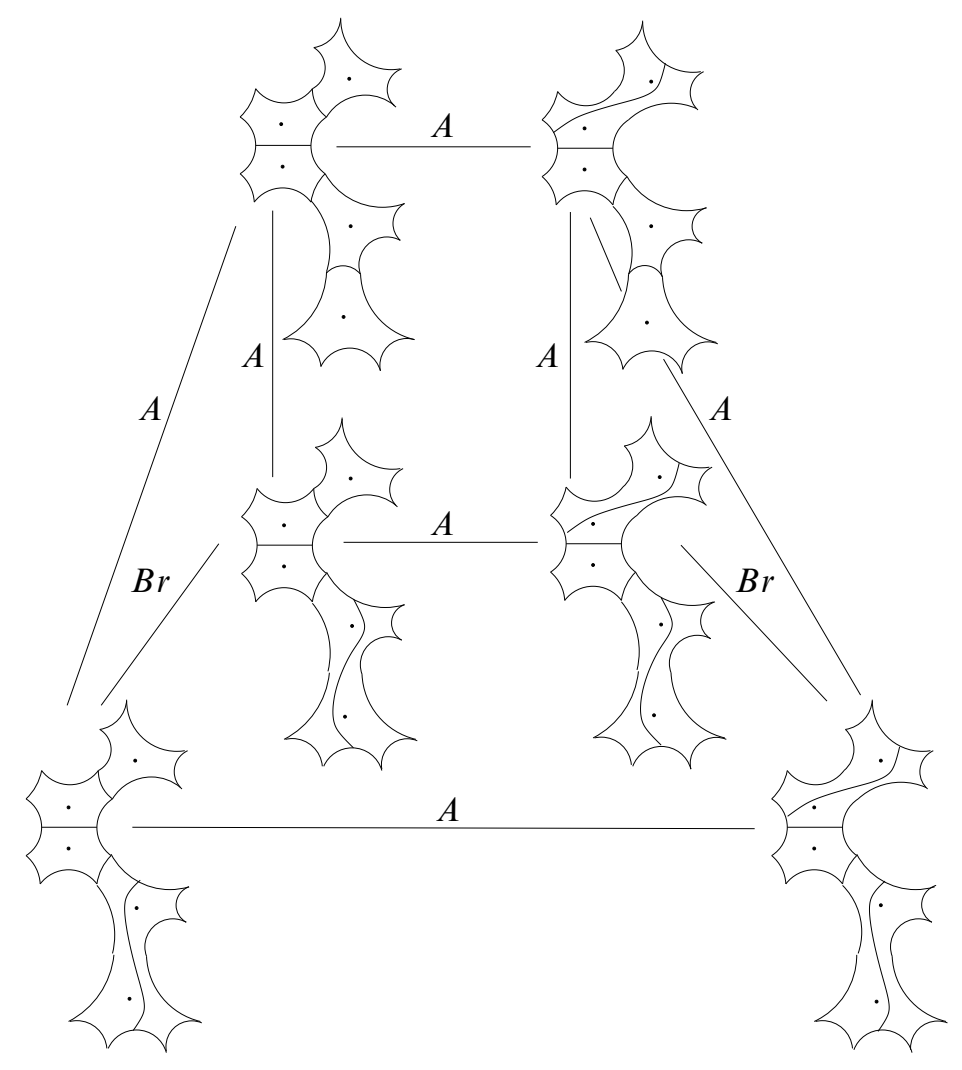

Figure 20: Relation between two inequivalent cycles of type $D C_{2}$, bis

The second lemma to prove is:

Lemma 4.3 Each square cycle of the form $A_{1} A_{2}=A_{2} A_{1}$ in the 1-skeleton of $\mathcal{C}^{+}\left(T^{\sharp}\right)$ or $\mathcal{C}\left(T^{\sharp}\right)$, resulting from by the commutation of two $A$-moves along disjoint arcs of a rigid structure, may be filled in by 2 -cells of $\mathcal{C}^{+}\left(T^{\sharp}\right)$, of $T$-type $D C_{1}, D C_{2}$, or of pentagonal $T$-type. Therefore, by Lemma 4.2, it may be filled in by 2 -cells which all belong to $\mathcal{C}\left(T^{\sharp}\right)$, hence is homotopically trivial in $\mathcal{C}\left(T^{\sharp}\right)$.

Proof Let $\gamma$ denote the square cycle $A_{1} A_{2}=A_{2} A_{1}$ in $\mathcal{C}^{+}\left(T^{\sharp}\right)$ or $\mathcal{C}\left(T^{\sharp}\right)$. In [22], it is proved that the square $f(\gamma)$ in $\mathcal{H} \mathcal{T}_{\text {red }}(D)$ may be filled in by 2-cells $\left(D C_{1}, D C_{2}\right.$ and pentagons). Let us enumerate them by $\omega_{1}, \ldots \omega_{n}$ in such a way that $\omega_{i}$ and $\omega_{i+1}$ (for $i=1, \ldots, n-1$ ) are adjacent along an edge, as well as $\omega_{n}$ and $\omega_{1}$. Following this enumeration, one may lift each $\omega_{i}$ to a $2-$ cell $\widetilde{\omega_{i}}$ of $\mathcal{C}^{+}\left(T^{\sharp}\right)$, in such a way that the $n$ lifts fill in the cycle $\gamma$. 


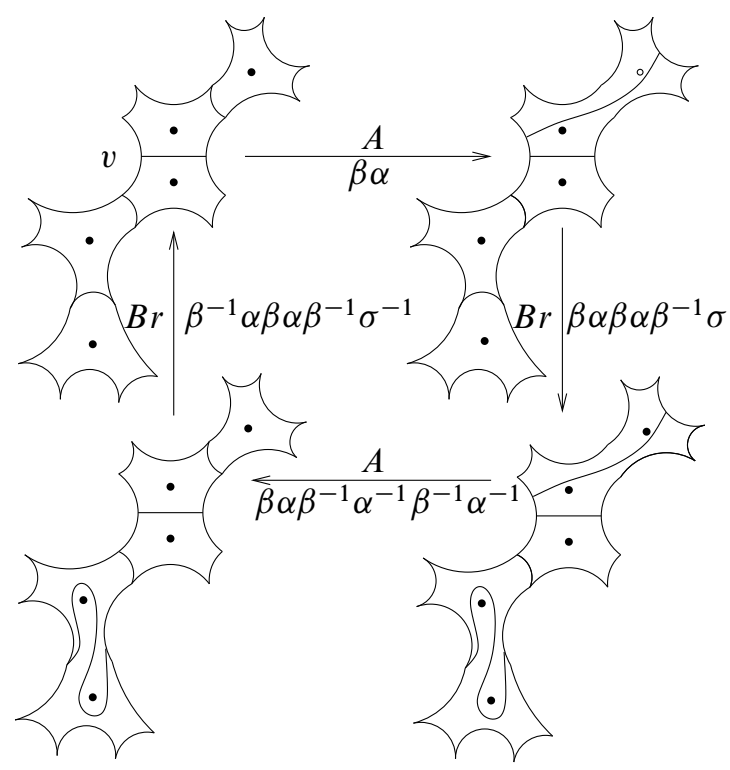

Figure 21: First cycle $B r A=A B r$ of level 7

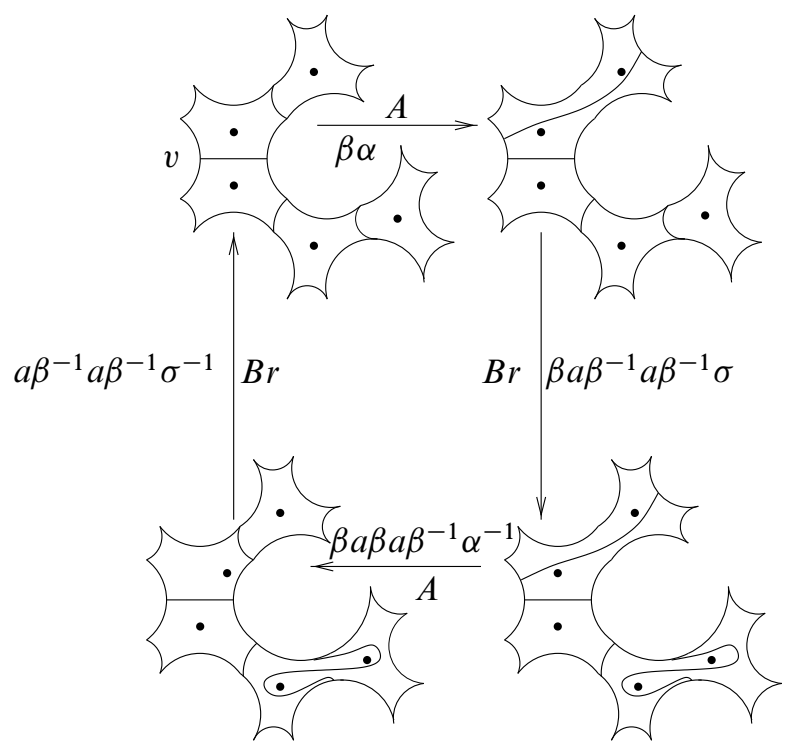

Figure 22: Second cycle $B r A=A B r$ of level 7 


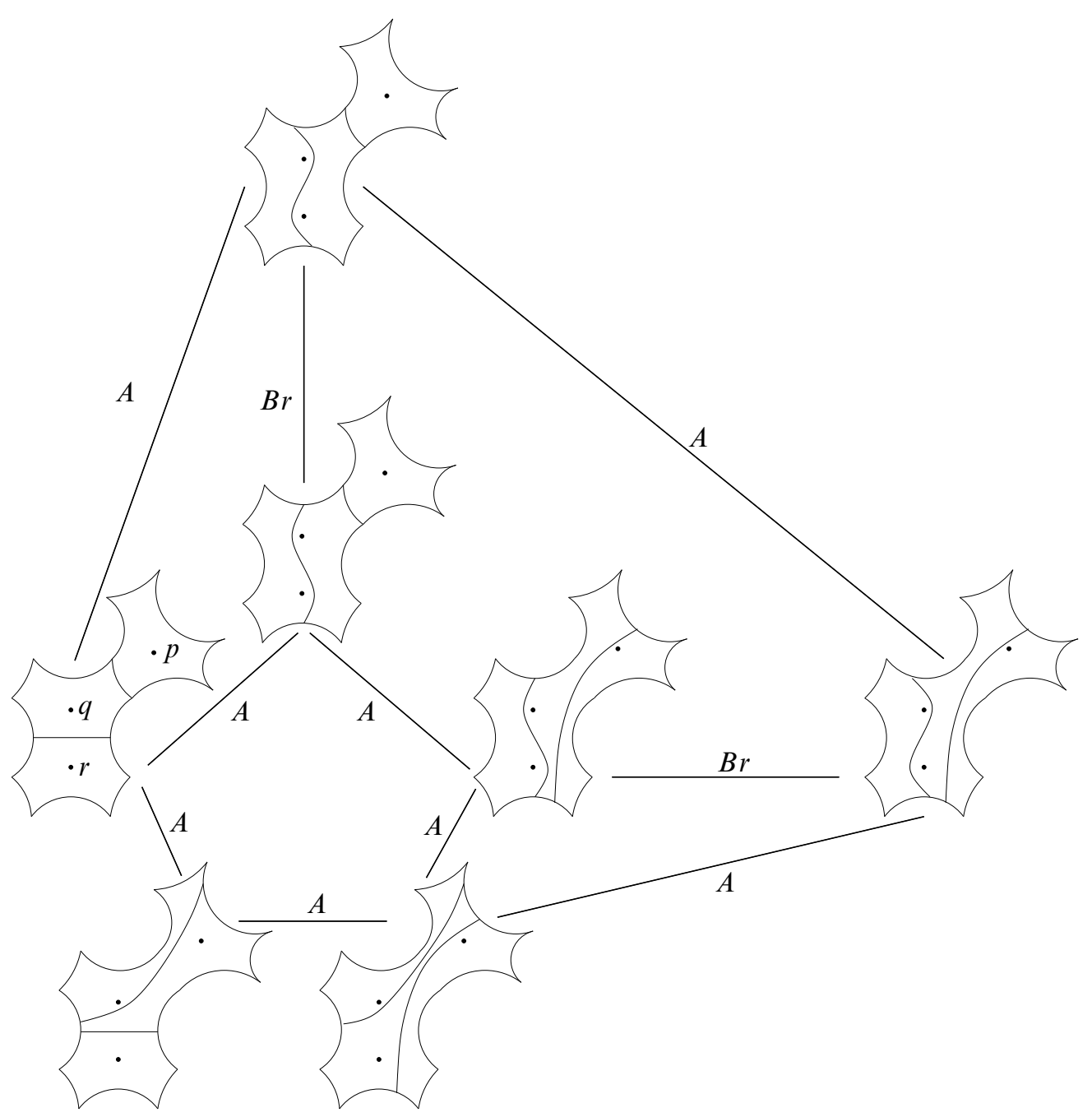

Figure 23: Relation between two inequivalent pentagonal cycles

The third and last lemma is:

Lemma 4.4 The square cycles $B r_{1} B r_{2}=B r_{2} B r_{1}$ and $A B r=B r A$, bounding the 2-cells of $\mathcal{C}^{+}\left(T^{\sharp}\right)$ which are not in $\mathcal{C}\left(T^{\sharp}\right)$, are filled in by some of 2-cells belonging to $\mathcal{C}\left(T^{\sharp}\right)$. Hence they are homotopically trivial in $\mathcal{C}\left(T^{\sharp}\right)$.

Proof The key point is that a $B r$-move may be seen as the composite of two $A-$ moves ( $A A=B r$ ), so that each relation of commutation involving $B r$-moves reduces 
to relations involving $A$-moves. Figure 24 shows how the square cycles $B r_{1} B r_{2}=$ $B r_{2} B r_{1}$ are filled in by 4 squares $A_{1} A_{2}=A_{2} A_{1}$ and four triangles $A A=B r$. Since each square $A_{1} A_{2}=A_{2} A_{1}$ is filled in by cells belonging to $\mathcal{C}\left(T^{\sharp}\right)$ by Lemma 4.3, this proves our claim. Similarly, Figure 25 shows how the square cycles $A B r=B r A$ are filled in by 2 cells of type $A A=B r$ and 2 squares $A_{1} A_{2}=A_{2} A_{1}$.

Since the complement of $\mathcal{C}\left(T^{\sharp}\right)$ in $\mathcal{C}^{+}\left(T^{\sharp}\right)$ is a union of cells $B r_{1} B r_{2}=B r_{2} B r_{1}$ and $A B r=B r A$, the last Lemma implies that the inclusion $\mathcal{C}\left(T^{\sharp}\right) \subset \mathcal{C}^{+}\left(T^{\sharp}\right)$ induces an isomorphism at the $\pi_{1}$ level. This ends the proof of Proposition 4.1.

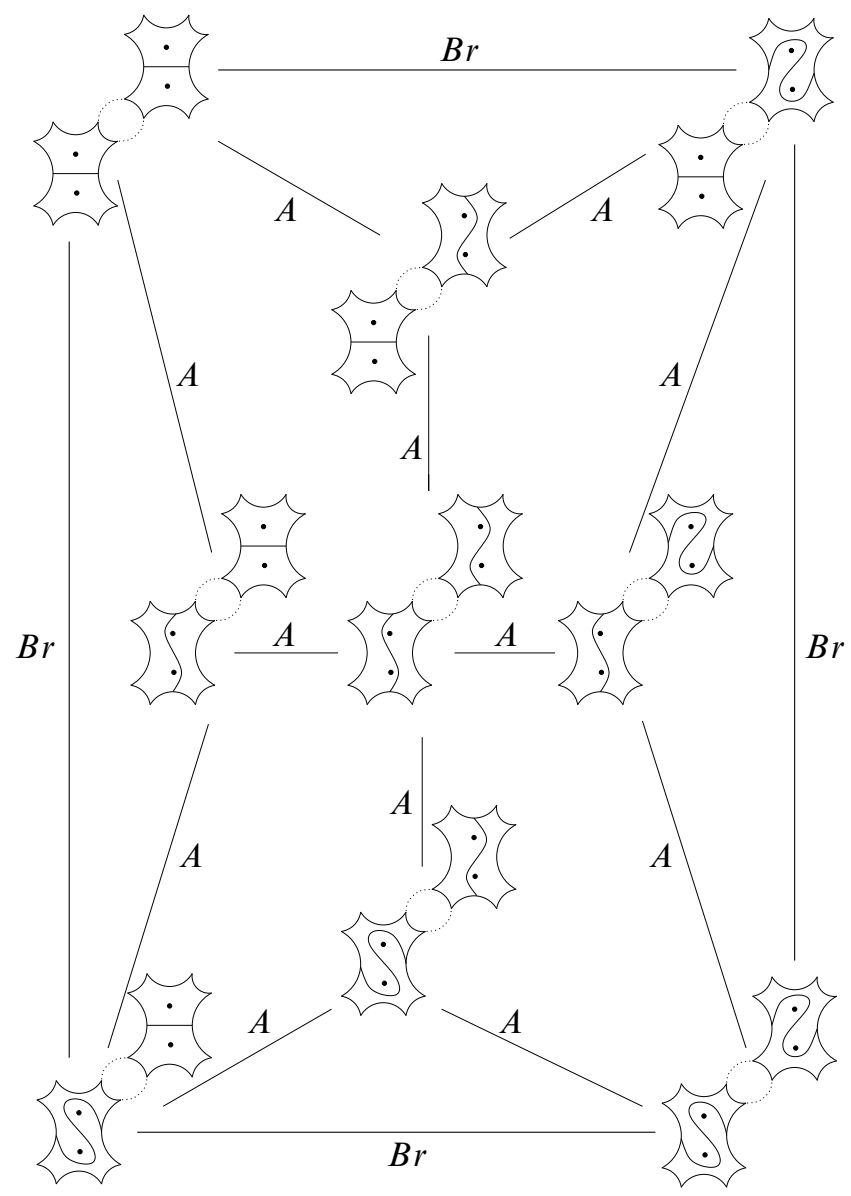

Figure 24: A cycle $B r_{1} B r_{2}=B r_{2} B r_{1}$ is filled in by cells of types $A_{1} A_{2}=$ $A_{2} A_{1}$ and $A A=B r$ 


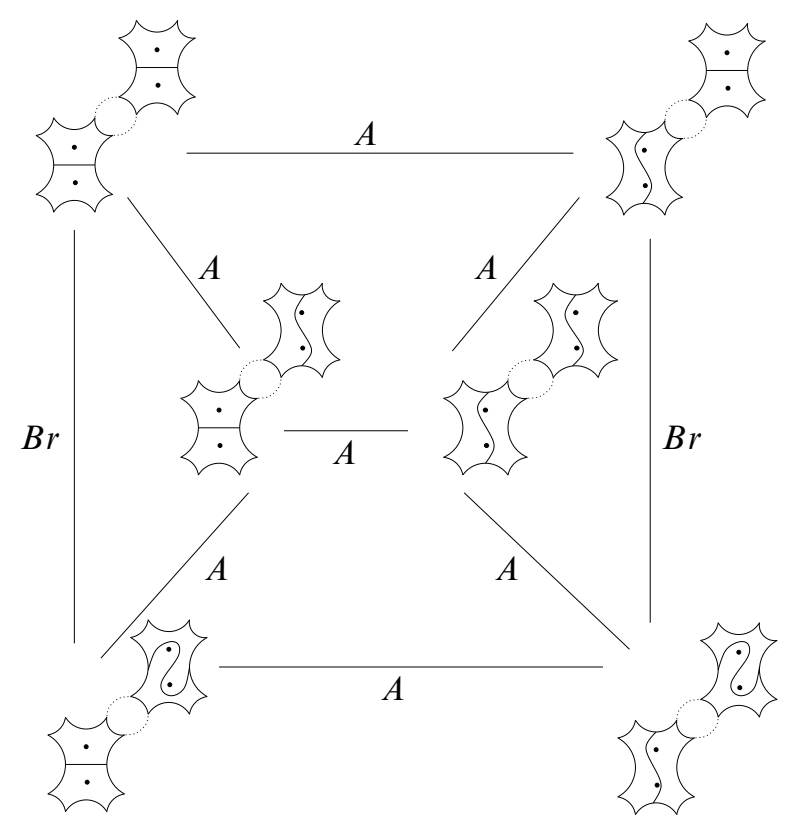

Figure 25: A cycle $A B r=B r A$ is filled in by cells of types $A_{1} A_{2}=A_{2} A_{1}$ and $A A=B r$

Theorem 4.5 The group $T^{\sharp}$ is finitely presented.

Proof The group $T^{\sharp}$ acts cocompactly on the simply connected complex $\mathcal{C}\left(T^{\sharp}\right)$. The stabilizers of the vertices are all isomorphic to $\operatorname{PSL}(2, \mathbb{Z})$. Indeed, since all vertices are equivalent modulo $T^{\sharp}$, it suffices to consider the case of the canonical rigid structure $v=\mathfrak{r}^{\#}$. Clearly, the stabilizer of $\mathfrak{r}^{\#}$ is also the group of orientationpreserving automorphisms of the tree of $D^{\sharp}$. It is isomorphic to $\operatorname{PSL}(2, \mathbb{Z})$ (see also Remark 2.3).

We claim that the stabilizers of the edges are isomorphic to $\mathbb{Z} / 2 \mathbb{Z}$. Indeed, there are two distinct classes of edges (modulo $T^{\sharp}$ ), which may be represented by two edges $e_{1}$ and $e_{2}$, based at the same origin $v$. The edge $e_{1}$ corresponds to an $A$-move on an arc of reference $\gamma$, while $e_{2}$ corresponds to a $B r$-move on the same arc $\gamma$. We may assume that the mapping class $\alpha^{\sharp} \in T^{\#}$ of Section 2.2 has been chosen such that the terminal vertex of $e_{1}$ is $\alpha^{\sharp}(v)$. Recall that it is a rigid rotation of order 4 outside $H_{1} \cup H_{2}$, but it fixes $q_{1}$ and $q_{2}$ inside $H_{1} \cup H_{2}$; see Figure 26 .

We denote by $\sigma \in B_{\infty} \subset T^{\sharp}$ the positive braiding on the arc $\gamma$. It permutes $q_{1}$ and $q_{2}$ and is such that $\sigma(v)$ is the terminal vertex of $e_{2}$; see Figure 26. 


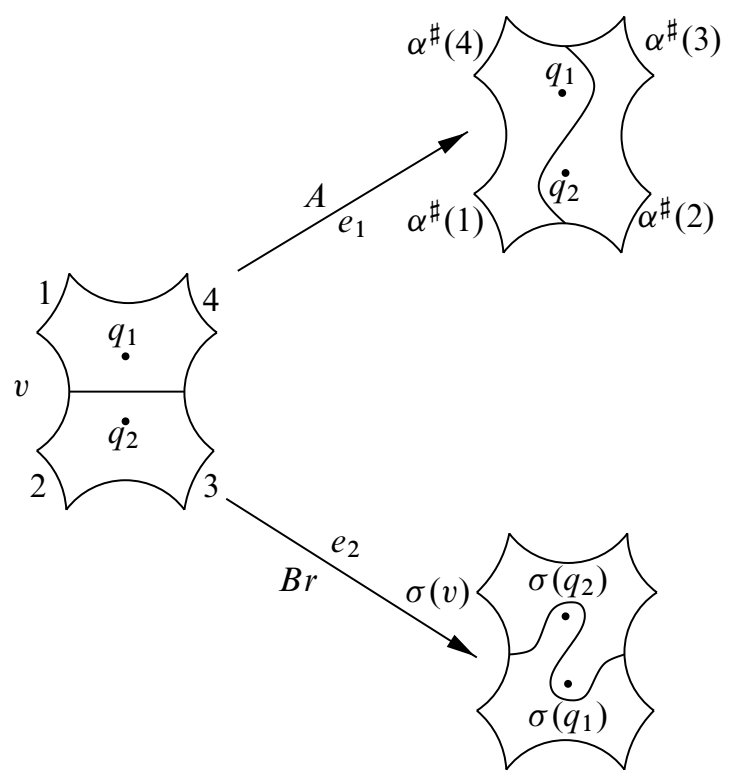

Figure 26: Edges $e_{1}$ and $e_{2}$ and generators $\alpha^{\sharp}$ and $\sigma$

One first checks that there is no element of $T^{\sharp}$ that reverses the orientation of the edges $e_{1}$ or $e_{2}$. Thus, the stabilizers $T_{e_{1}}^{\#}$ of $e_{1}$ and $T_{e_{2}}^{\#}$ of $e_{2}$ are subgroups of the stabilizer of $v$. In fact, $T_{e_{1}}^{\#}=T_{e_{2}}^{\#}$, generated by the element $a \in P S L(2, \mathbb{Z})$ of order 2 , which is rigid rotation of angle $\pi$ that interchanges the hexagons $H_{1}$ and $H_{2}$ and preserves the arc $\gamma$ (reversing its orientation). We shall see below that $a=\sigma^{-1} \alpha^{\sharp 2}=\alpha^{\sharp 2} \sigma^{-1}$ (beware that $\alpha^{\sharp 2}$ is not of order 2!).

Since the stabilizers of the vertices are finitely presented and the stabilizers of the edges are finitely generated, Theorem 1 of [7] asserts that $T^{\#}$ is finitely presented.

Remark 4.6 The stabilizer $T_{v}^{\#}$ of $v$ admits the following presentation:

$$
T_{v}^{\sharp}=\left\langle a, \beta^{\sharp} \mid a^{2}=\beta^{\sharp 3}=1\right\rangle \cong \operatorname{PSL}(2, \mathbb{Z}) .
$$

\subsection{A presentation for $T^{\sharp}$}

\subsubsection{Statement of the theorem}

Theorem 4.7 The group $T^{\sharp}$ admits a finite presentation, with three generators $\alpha^{\sharp}, \beta^{\sharp}$ and $\sigma$, and the following relations: 
(1) $\alpha^{\sharp} \sigma=\sigma \alpha^{\sharp}$

(2) $\alpha^{\sharp 4}=\sigma^{2}$

(3) $\beta^{\sharp 3}=1$

(4) $\left(\beta^{\sharp} \alpha^{\sharp}\right)^{5}=\sigma \beta^{\sharp} \sigma \beta^{\sharp-1} \sigma$

Setting $a=\alpha^{\sharp 2} \sigma^{-1}$ :

(5) $\left[\beta^{\sharp} \alpha^{\sharp} \beta^{\sharp}, a \beta^{\sharp} \alpha^{\sharp} \beta^{\sharp} a\right]=1$ (level 5)

(6) $\left[\beta^{\sharp} \alpha^{\sharp} \beta^{\sharp}, a \beta^{\sharp 2} a \beta^{\sharp} \alpha^{\sharp} \beta^{\sharp} a \beta^{\sharp} a\right]=1$

Consistency relations:

(7) $\left[\sigma, \beta^{\sharp} \alpha^{\sharp} \beta^{\sharp}\right]=1$ (level 5)

(8) $\left[\sigma, \beta^{\sharp} \alpha^{\sharp} \beta^{\sharp} a \beta^{\sharp-1}\right]=1$ (level 6)

(9) $\left[\sigma, \beta^{\sharp} a \beta^{\sharp-1} a \beta^{\sharp} \alpha^{\sharp} \beta^{\sharp} a \beta^{\sharp} a \beta^{\sharp-1}\right]=1$ (level 7)

(10) $\left[\sigma, \beta^{\sharp} a \beta^{\sharp} a \beta^{\sharp} \alpha^{\sharp} \beta^{\sharp} a \beta^{\sharp} a \beta^{\sharp}\right]=1$ (level 7)

Commutations of braidings:

(11) $\left[\sigma, \beta^{\sharp} a \beta^{\sharp-1} \sigma\left(\beta^{\sharp} a \beta^{\sharp-1}\right)^{-1}\right]=1$ (level 6)

(12) $\left[\sigma, \beta^{\sharp} a \beta^{\sharp} a \beta^{\sharp-1} \sigma\left(\beta^{\sharp} a \beta^{\sharp} a \beta^{\sharp-1}\right)^{-1}\right]=1$ (level 7)

(13) $\left[\sigma, \beta^{\sharp} a \beta^{\sharp} a \beta^{\sharp} \sigma\left(\beta^{\sharp} a \beta^{\sharp} a \beta^{\sharp}\right)^{-1}\right]=1$ (level 7)

Setting $\sigma_{1}=\sigma, \sigma_{2}=\beta^{\sharp} \sigma \beta^{\sharp-1}$ and $\sigma_{3}=\beta^{\sharp-1} \sigma \beta^{\sharp}$ :

(14) $\sigma_{1} \sigma_{2} \sigma_{1}=\sigma_{2} \sigma_{1} \sigma_{2}$ (fundamental relation of the braid group)

(15) $\sigma_{1} \sigma_{2} \sigma_{3} \sigma_{1}=\sigma_{2} \sigma_{3} \sigma_{1} \sigma_{2}=\sigma_{3} \sigma_{1} \sigma_{2} \sigma_{3}$ (Sergiescu's relations)

Corollary 4.8 We have $H_{1}\left(T^{\sharp}\right)=\mathbb{Z} / 6 \mathbb{Z}$. In particular, the groups $T^{\sharp}$ and $T^{*}$ are not isomorphic.

Proof $H_{1}\left(T^{\sharp}\right)$ is generated by the commuting $\left[\alpha^{\sharp}\right],\left[\beta^{\sharp}\right]$ and $[\sigma]$, subject to the relations $4\left[\alpha^{\sharp}\right]=2[\sigma], 3\left[\beta^{\sharp}\right]=0$ and $5\left[\alpha^{\sharp}\right]+5\left[\beta^{\sharp}\right]=3[\sigma]$. They are equivalent to $\left[\alpha^{\sharp}\right]=-[\sigma],\left[\beta^{\sharp}\right]=-2[\sigma]$ and $6[\sigma]=0$, hence the claim.

4.2.2 Generators We follow the method described by K Brown in [7], derived from the Bass-Serre theory.

Recall that the quotient $\mathcal{C}\left(T^{\sharp}\right) / T^{\sharp}$ possesses a unique vertex, represented by $v$, and two edges, represented by $e_{1}$ and $e_{2}$ (see the proof of Lemma 3.3).

The stabilizers of $e_{1}$ and $e_{2}$ are of order 2, generated by $a$. For better clarity, we write $T_{e_{1}}^{\#}=\left\langle a_{1}, a_{1}^{2}=1\right\rangle$ and $T_{e_{2}}^{\#}=\left\langle a_{2}, a_{2}^{2}=1\right\rangle$, respectively. 
4.2.3 Relations Theorem 1 of [7] states that $T^{\#}$ is generated by the stabilizer $T_{v}^{\#}$ and by the elements $g_{e_{1}}=\alpha^{\#}$ and $g_{e_{2}}=\sigma$, subject to the following relations:

- Presentation (i). For each $e \in\left\{e_{1}, e_{2}\right\}, g_{e}^{-1} i_{e}(h) g_{e}=c_{e}(h)$ for all $h \in T_{e}^{\#}$, where $i_{e}$ is the inclusion $T_{e}^{\#} \hookrightarrow T_{v}^{\#}$ and $c_{e}: T_{e}^{\#} \rightarrow T_{v}^{\#}$ is the conjugation morphism $h \mapsto g_{e}^{-1} h g_{e}$.

Explicitly, $g_{e_{1}}^{-1} i_{e_{1}}(h) g_{e_{1}}=c_{e_{1}}(h)$ with $g_{e_{1}}=\alpha^{\#}$ and $h=a_{1} \in T_{e_{1}}^{\#}$ provides the relation $\alpha^{\sharp-1} a \alpha^{\sharp}=\alpha^{\sharp-1} a_{1} \alpha^{\sharp}$, where the right hand side is computed in $T_{v}^{\sharp}$, in which it is equal to $a$. Hence the relation

$$
\alpha^{\sharp-1} a \alpha^{\sharp}=a .
$$

As for $g_{e_{2}}^{-1} i_{e_{2}}(h) g_{e_{2}}=c_{e_{2}}(h)$ with $g_{e_{2}}=\sigma$ and $h=a_{2} \in T_{e_{2}}^{\#}$, it provides the relation $\sigma^{-1} a \sigma=\sigma^{-1} a_{2} \sigma$, where the right hand side is computed in $T_{v}^{\sharp}$, in which it is equal to $a$. Hence the relation

$$
\sigma^{-1} a \sigma=a .
$$

- Presentation (ii). $\quad r_{\tau}=1$ for each 2-cell $\tau \in \mathcal{C}\left(T^{\sharp}\right)$, where $r_{\tau}$ is a word in the generators of $T_{v}^{\#}, \alpha^{\#}$ and $\sigma$, associated with the 2-cell $\tau$ in the way described in [7]. We recall it for the convenience of the reader:

Each edge of the complex starting at $v$ has one of the following forms:

(1) $v \longrightarrow\left(h \alpha^{\sharp \pm 1}\right)(v), h \in T_{v}^{\#}$

(2) $v \longrightarrow\left(h \sigma^{ \pm 1}\right)(v), h \in T_{v}^{\#}$

To such an edge $e$ we associate an element $\gamma \in T^{\sharp}$ such that $e$ ends at $\gamma(v): \gamma=h \alpha^{\sharp \pm 1}$ in case (a), $\gamma=h \sigma^{ \pm 1}$ in case (b).

Let $\tau$ be one of the 2-cells of the complex $\mathcal{C}\left(T^{\sharp}\right)$. One chooses an orientation and a cyclic labeling of the boundary edges, such that the labeled 1 edge $E_{1}$ starts from the vertex $v$.

Let $\gamma_{1}$ be associated to $E_{1}$ as above. It ends at $\gamma_{1}(v)$, so the second edge is of the form $\gamma_{1}\left(E_{2}\right)$ for some edge $E_{2}$ starting at $v$. Let $\gamma_{2}$ be associated to $E_{2}$. The second edge ends at $\gamma_{1} \gamma_{2}(v)$. If $n$ is the length of the cycle bounding $\tau$, one obtains this way a sequence $\gamma_{1}, \ldots, \gamma_{n}$ such that $\gamma_{1} \cdots \gamma_{n}(v)=v$.

Note that for each of the cycles, we have indicated the corresponding $\gamma_{i}$ above the $i$-th edge. 
Let $\gamma$ be the element of the stabilizer $T_{v}^{\#}$ which is equal to $\gamma_{1} \cdots \gamma_{n}$ when each element $\gamma_{i}$ is viewed in $T^{\#}$. Then the relation associated to $\tau$ is

$$
\gamma_{1} \cdots \gamma_{n}=\gamma
$$

where the left hand side is viewed as a word in $\alpha^{\sharp}, \sigma, a, \beta^{\sharp}$, and their inverses.

Following this process for the $2-$ cells of the complex $\mathcal{C}\left(T^{\sharp}\right)$, one obtains:

(1) Cell $A A=B r$ (Figure 9). The corresponding relation is $\alpha^{\sharp} \sigma^{-1} \alpha^{\sharp}=a$. Equivalently, $\sigma=\alpha^{\sharp} a^{-1} \alpha^{\sharp}$. Since by (*), $\alpha^{\sharp}$ and $a$ commute, one obtains $\sigma=\alpha^{\sharp 2} a^{-1}$, hence $\alpha^{\sharp}$ and $\sigma$ commute (Relation 1). Since $a=\sigma^{-1} \alpha^{\sharp 2}, a$ may be eliminated, and the relation $a^{2}=1$ is now equivalent to $\alpha^{\sharp 4}=\sigma^{2}$ (Relation 2).

(2) (a) Cell $D C_{1}$ (Figure 10). The corresponding relation is

$$
\left[\beta^{\sharp} \alpha^{\sharp} \beta^{\sharp}, a \beta^{\sharp} \alpha^{\sharp} \beta^{\sharp} a\right]=1 \text { (Relation 5). }
$$

(b) Cell $\mathrm{DC}_{2}$ (Figure 11). The corresponding relation is

$$
\left[\beta^{\sharp} \alpha^{\sharp} \beta^{\sharp}, a \beta^{\sharp 2} a \beta^{\sharp} \alpha^{\sharp} \beta^{\sharp} a \beta^{\sharp} a\right]=1 \text { (Relation 6). }
$$

(c) Pentagonal cell (Figure 12). It gives first the relation

$$
\alpha^{\sharp-1} \beta^{\sharp-1} \alpha^{\sharp-1} \beta^{\sharp-1} \alpha^{\sharp-1} \sigma \beta^{\sharp-1} \alpha^{\sharp-1} \sigma \beta^{\sharp-1} \alpha^{\sharp}=\beta^{\sharp} a .
$$

Taking the inverse of this relation, one obtains

$$
\alpha^{\sharp-1} \beta^{\sharp} \sigma^{-1} \alpha^{\sharp} \beta^{\sharp} \sigma^{-1} \alpha^{\sharp} \beta^{\sharp} \alpha^{\sharp} \beta^{\sharp} \alpha^{\sharp}=a \beta^{\sharp-1} .
$$

Replacing $a$ by $a=\alpha^{\sharp-2} \sigma$, one obtains $\alpha^{\sharp} \beta^{\sharp} \sigma^{-1} \alpha^{\sharp} \beta^{\sharp} \sigma^{-1}\left(\alpha^{\sharp} \beta^{\sharp}\right)^{3}=$ $\sigma$. Equivalently, $\sigma^{-1} \alpha^{\sharp} \beta^{\sharp} \sigma^{-1}\left(\alpha^{\sharp} \beta^{\sharp}\right)^{4}=\left(\alpha^{\sharp} \beta^{\sharp}\right)^{-1} \sigma \alpha^{\sharp} \beta^{\sharp}$. Since $\alpha^{\sharp}$ and $\sigma$ commute, the right hand side is equal to $\beta^{\sharp-1} \sigma \beta^{\sharp}$. Hence the relation $\sigma^{-1} \alpha^{\sharp} \beta^{\sharp} \sigma^{-1}\left(\alpha^{\sharp} \beta^{\sharp}\right)^{-1}\left(\alpha^{\sharp} \beta^{\sharp}\right)^{5}=\beta^{\sharp-1} \sigma \beta^{\sharp}$. This is equivalent to $\left(\beta^{\sharp} \alpha^{\sharp}\right)^{5}=\beta^{\sharp} \sigma \beta^{\sharp-1} \alpha^{\sharp-1} \sigma \beta^{\sharp-1} \sigma \beta^{\sharp} \alpha^{\sharp}$. Using once again the commutation between $\sigma$ and $\alpha^{\sharp}$, one obtains $\left(\beta^{\sharp} \alpha^{\sharp}\right)^{5}=\beta^{\sharp} \sigma \beta^{\sharp-1} \sigma \alpha^{\sharp-1} \beta^{\sharp-1} \sigma \beta^{\sharp} \alpha^{\sharp}$. But we shall see below that $\sigma$ and $\beta^{\sharp} \alpha^{\sharp} \beta^{\sharp}$ commute (see Cell $A B r=B r A$ of level 5), so that $\alpha^{\sharp-1} \beta^{\sharp-1} \sigma \beta^{\sharp} \alpha^{\sharp}=\beta^{\sharp} \sigma \beta^{\sharp-1}$. Finally, the relation becomes

$$
\left(\beta^{\sharp} \alpha^{\sharp}\right)^{5}=\beta^{\sharp} \sigma \beta^{\sharp-1} \sigma \beta^{\sharp} \sigma \beta^{\sharp-1} \text {. }
$$

Modulo the braid relation (see below Cells coming from the presentation of the braid group), this is Relation 4.

(3) (a) Cell $A B r=B r A$ of level 5 (Figure 16). $\left[\sigma, \beta^{\sharp} \alpha^{\sharp} \beta^{\sharp}\right]=1$ (Relation 7)

(b) Cell $A B r=B r A$ of level 6 (Figure 18). $\left[\sigma, \beta^{\sharp} \alpha^{\sharp} \beta^{\sharp} a \beta^{\sharp-1}\right]=1$ (Relation 8) 
(c) First cell $A B r=B r A$ of level 7 (Figure 21).

$$
\left[\sigma, \beta^{\sharp} a \beta^{\sharp-1} a \beta^{\sharp} \alpha^{\sharp} \beta^{\sharp} a \beta^{\sharp} a \beta^{\sharp-1}\right]=1 \text { (Relation 9) }
$$

(d) Second cell $A B r=B r A$ of level 7 (Figure 22).

$$
\left[\sigma, \beta^{\sharp} a \beta^{\sharp} a \beta^{\sharp} \alpha^{\sharp} \beta^{\sharp} a \beta^{\sharp} a \beta^{\sharp}\right]=1 \text { (Relation 10) }
$$

(4) (a) Cell $\mathrm{Br}_{1} \mathrm{Br}_{2}=\mathrm{Br}_{2} B r_{1}$ of level 6 (Figure 13).

$$
\left[\sigma, \beta^{\sharp} a \beta^{\sharp-1} \sigma\left(\beta^{\sharp} a \beta^{\sharp-1}\right)^{-1}\right]=1 \text { (Relation } 11 \text { ) }
$$

(b) First cell $\mathrm{Br}_{1} \mathrm{Br}_{2}=\mathrm{Br}_{2} B r_{1}$ of level 7 (Figure 14).

$$
\left[\sigma, \beta^{\sharp} a \beta^{\sharp} a \beta^{\sharp-1} \sigma\left(\beta^{\sharp} a \beta^{\sharp} a \beta^{\sharp-1}\right)^{-1}\right]=1 \text { (Relation 12) }
$$

(c) Second cell $B r_{1} B r_{2}=B r_{2} B r_{1}$ of level 7 (Figure 15).

$$
\left[\sigma, \beta^{\sharp} a \beta^{\sharp} a \beta^{\sharp} \sigma\left(\beta^{\sharp} a \beta^{\sharp} a \beta^{\sharp}\right)^{-1}\right]=1 \text { (Relation 13) }
$$

(5) Cells coming from the presentation of the braid group. They obviously give Relation 14 and Relation 15.

\section{3 $\quad T^{*}$ is finitely presented}

The groups $T^{\#}$ and $T^{*}$, though both alike, are not isomorphic. However, there is a proof for the assertion that $T^{*}$ is finitely presented which mimics that for $T^{\sharp}$. One introduces $T^{*}$-complexes $\mathcal{C}^{+}\left(T^{*}\right)$ and $\mathcal{C}\left(T^{*}\right)$, whose vertices are the asymptotically rigid structures of $D^{*}$, and the edges are of two types, corresponding to moves $A$ and $\mathrm{Br}$.

- If $\mathfrak{r}$ is an asymptotically rigid structure and $\gamma$ is an arc of $\mathfrak{r}$, the $A$-move on $\gamma$ keeps unchanged all the arcs of $\mathfrak{r}$ except $\gamma$, and replaces $\gamma$ by $\gamma^{\prime}$ which is transverse to $\gamma$ and passes through the same puncture as $\gamma$ (see Figure 27). Note that there is a unique $A$-move on $\gamma$.

- If $\mathfrak{r}$ is an asymptotically rigid structure and $p$ and $q$ are two punctures of $D^{*}$ on two different sides of a hexagon $H$ of $\mathfrak{r}$, there is a simple arc $e$ inside $H$ which connects $p$ to $q$. Let $\sigma_{e}$ be the braiding along $e$. The move $B r$ changes $\mathfrak{r}$ by the natural action of $\sigma_{e}$ on $\mathfrak{r}$ (see Figure 27).

The complex $\mathcal{C}^{+}\left(T^{*}\right)$ has the same types of 2 -cells as $\mathcal{C}^{+}\left(T^{\sharp}\right)$, except that the cell $A A=B r$ of $\mathcal{C}^{+}\left(T^{\sharp}\right)$ does not exist, and the pentagonal cell is replaced by a hexagonal cell, expressing that a certain sequence of five $A$-moves produces the effect of a 


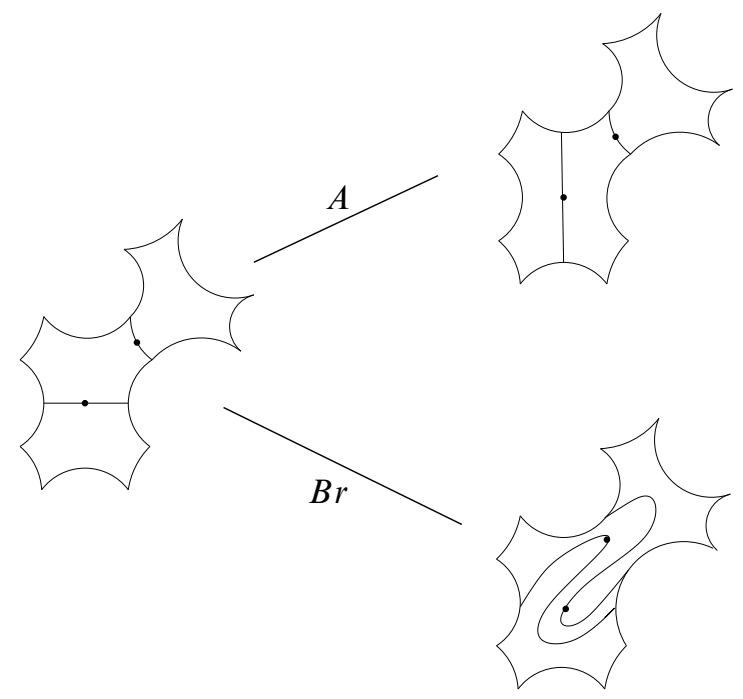

Figure 27: The two types of edges in $\mathcal{C}\left(T^{*}\right)$

$B r$-move; see Figure 28. As can be guessed, the relation which will be associated to this cell is $\left(\beta^{*} \alpha^{*}\right)^{5}=\sigma_{[04]}$; see Lemma 2.6.

The Cayley complex of $B_{\infty}$, for the Sergiescu presentation associated to the graph of $D^{*}$ (see Remark 2.7), provides 2-cells of $\mathcal{C}\left(T^{*}\right)$ which involve only $B r$-moves. Among them are cells of commutation $B r_{1} B r_{2}=B r_{2} B r_{1}$, which provide countably many $T^{*}$-types of cells (that is, countably many cells in the quotient $\mathcal{C}\left(T^{*}\right) / T^{*}$ ). On the other hand, the cells of the Cayley complex coming from the other types of relations (that is, the noncommuting types) provide only finitely many cells in $\left.\mathcal{C}\left(T^{*}\right) / T^{*}\right)$. This is due to the regularity of the graph from which the presentation of $B_{\infty}$ is derived. Therefore, just like $\mathcal{C}\left(T^{\sharp}\right) / T^{\sharp}$, the quotient $\mathcal{C}\left(T^{*}\right) / T^{*}$ is made of countably many 2-cells $B r_{1} B r_{2}=B r_{2} B r_{1}$ and $A B r=B r A$, plus finitely many other 2-cells.

Fortunately, the key role played by the 2-cell $A A=B r$ in the proof of Theorem 4.5, especially in Lemma 4.4, is now played by the 2-cell $A^{5}=B r$ : one eliminates almost all the $T^{*}$-types of 2-cells $A B r=B r A$ and $B r_{1} B r_{2}=B r_{2} B r_{1}$ using cycles $A_{1} A_{2}=A_{2} A_{1}$ (the analogue of Lemma 4.3 is true) and $B r=A^{5}$. This enables us to obtain a reduced complex $\mathcal{C}\left(T^{*}\right)$ which is finite modulo $T^{*}$ and simply connected. These arguments constitute a sketch of the proof of the following theorem:

Theorem 4.9 The braided Ptolemy-Thompson group $T^{*}$ is finitely presented and admits a presentation with 2 generators. 


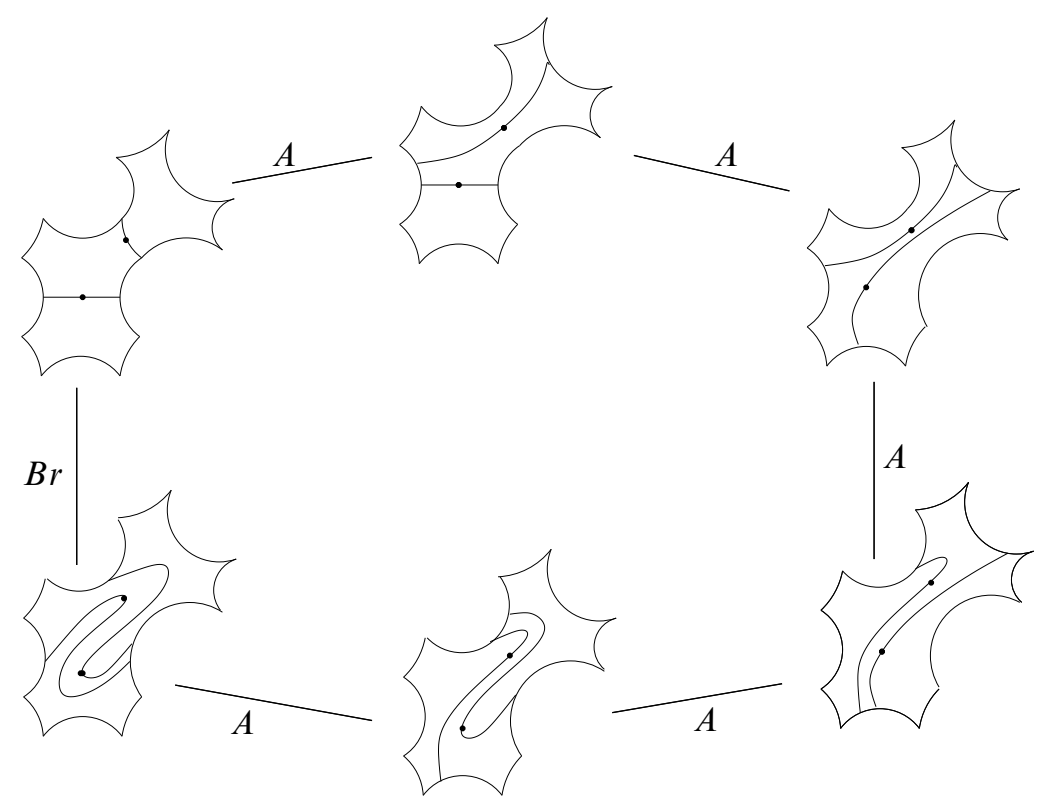

Figure 28: Relation $A^{5}=B r$

\section{Comments and open questions}

Actions by homeomorphisms on $S^{1} \quad$ D Calegari [9] proved that punctured mapping class groups have a faithful action by homeomorphisms on $S^{1}$. Specifically, let $S$ be a surface (possibly of infinite type) with a base point $p$. Let $\operatorname{Mod}(S, p)$ be the group $\mathrm{Homeo}^{+}(S, p) / \mathrm{Homeo}_{0}^{+}(S, p)$. Here $\mathrm{Homeo}^{+}(S, p)$ denotes the group of orientation-preserving homeomorphisms of $S$ to itself which takes $p$ to itself, and Homeo $_{0}^{+}(S, p)$ denotes the connected subgroup containing the identity map. Then $\operatorname{Mod}_{S, p}$ is circularly orderable. Notice that this punctured mapping class groups fits into an exact sequence

$$
1 \rightarrow \pi_{1}(S) \rightarrow \operatorname{Mod}(S, p) \rightarrow \operatorname{Mod}(S) \rightarrow 1
$$

where $\operatorname{Mod}(S)$ is the usual mapping class group of $S$.

In particular, there are extensions by free groups of the universal mapping class groups $\mathcal{B}$ in genus zero, which embed in Homeo ${ }_{+}\left(S^{1}\right)$, being circularly orderable. It seems, however, that $\mathcal{B}$ does not act faithfully on the circle.

Smoothing the action It is presently unknown whether the group $T^{\sharp}$ admits an embedding into some group of piecewise linear homeomorphisms $P L_{\lambda}\left(S^{1}\right)$ with 
break points and derivatives of the form $\lambda^{n}$, where $n \in \mathbb{Z}$. We conjecture that there is no embedding $T^{\sharp} \rightarrow \operatorname{Diff}_{+}^{2}\left(S^{1}\right)$ into the group of diffeomorphisms of $S^{1}$ of class $\mathcal{C}^{2}$. Specifically, any homomorphism $T^{\#} \rightarrow \operatorname{Diff}_{+}^{2}\left(S^{1}\right)$ should factor through a finite extension of $T$.

Automatic groups Thompson groups are known to be asynchronous automatic groups [26], but it is still unknown whether they are (synchronously) automatic. V Guba proved that the Dehn function of $F$ is quadratic, as is the case for all automatic groups. We conjecture that $T^{\#}$ is automatic. Notice that braid groups and more generally mapping class groups are known to be automatic [17; 34]. In particular, this would immediately imply that $T^{\#}$ is finitely presented and has solvable word problem. We expect that the conjugacy problem is solvable too, though it is presently unknown whether this holds true for all automatic groups. Moreover, automatic groups are combable [17, page 84] and hence they are $\mathrm{FP}_{\infty}$ and thus $\mathrm{F}_{\infty}$, ie they have a classifying space with finitely many cells in each dimension [17, page 220]. Eventually, the Dehn function of $T^{\sharp}$ should be quadratic. If $T^{\#}$ is biautomatic then it would provide an example of such a group having a free abelian subgroup of infinite rank.

Outer automorphisms groups It was first established by Dyer and Grossman that $\operatorname{Out}\left(B_{n}\right)=\mathbb{Z} / 2 \mathbb{Z}$, for $n \geq 4$, and Ivanov and further McCarthy extended this to mapping class groups. However, their result does not extend, as stated, to infinite braid groups. In fact there exists an embedding

$$
T \rightarrow \operatorname{Out}\left(B_{\infty}\right)
$$

induced by the action of $T^{\#}$ by conjugacy on its normal subgroup $B_{\infty}$. In particular, $\operatorname{Out}\left(B_{\infty}\right)$ seems to be a quite rich group.

On the other hand $\mathrm{M}$ Brin [6] proved that group $\operatorname{Out}(T)=\mathbb{Z} / 2 \mathbb{Z}$. It would be interesting to know whether $\operatorname{Out}\left(T^{\sharp}\right)=\mathbb{Z} / 2 \mathbb{Z}$ holds.

Remarks 5.1 (1) Any diagram group (see Guba and Sapir [27]) can be embedded into $B_{\infty}$ (by a result of Wiest [39]) and thus into $T^{\sharp}$. However, $T^{\#}$ is not a diagram group since it has torsion. Moreover, $T^{\#}$ and the Brin-Dehornoy braided Thompson group $B V$ are the typical examples of some more general braided diagram groups. The work of Farley on diagram groups extends to braided diagrams and pictures [18]. In particular, each one of these groups acts properly cellularly on a $C A T(0)$-complex, which is not locally finite. The stabilizers of cells are isomorphic to braid groups (on finitely many strands). One might expect these braided diagram groups to be finitely presented and even $\mathrm{FP}_{\infty}$ 
(2) The group $T^{\sharp}$ has not the Kazhdan property since $T$ has not.

(3) If $\Gamma$ is a lattice in a simple Lie group of and the $\mathbb{Q}$-rank of $\Gamma$ is at least 2 then any homomorphism $\Gamma \rightarrow T^{\#}$ should be trivial, since any $\mathcal{C}^{0}$-action of such a $\Gamma$ on $S^{1}$ is trivial, by a result of D Witte [40].

(4) There exist however homomorphisms from arithmetic groups of rank one into $T^{\sharp}$. In fact, Kontsevich and Soibelman recently constructed in [29] faithful homomorphisms from an arithmetic subgroup of $S O(1,18)$ into the braid groups.

(5) The group $T^{\sharp}$ is nonamenable and hence of exponential growth.

\section{References}

[1] E Artin, Theory of braids, Ann. of Math. (2) 48 (1947) 101-126 MR0019087

[2] B Bakalov, A Kirillov, Jr, On the lego-Teichmüller game, Transform. Groups 5 (2000) 207-244 MR1780935

[3] V N Bezverkhniĭ, Solution of the generalized conjugacy problem for words in $C(p) \& T(q)$-groups, Izv. Tul. Gos. Univ. Ser. Mat. Mekh. Inform. 4 (1998) 5-13 MR1751577

[4] J Birman, K H Ko, S J Lee, A new approach to the word and conjugacy problems in the braid groups, Adv. Math. 139 (1998) 322-353 MR1654165

[5] M G Brin, The Algebra of Strand Splitting. I. A Braided Version of Thompson's Group $V$ arXiv:math.GR/0406042

[6] M G Brin, The chameleon groups of Richard J. Thompson: automorphisms and dynamics, Inst. Hautes Études Sci. Publ. Math. (1996) 5-33 (1997) MR1441005

[7] K S Brown, Presentations for groups acting on simply-connected complexes, J. Pure Appl. Algebra 32 (1984) 1-10 MR739633

[8] K-U Bux, Braiding and tangling the chessboard complex arXiv:math.GT/0310420

[9] D Calegari, Circular groups, planar groups, and the Euler class, from: "Proceedings of the Casson Fest", Geom. Topol. Monogr. 7, Geom. Topol. Publ., Coventry (2004) 431-491 MR2172491

[10] J W Cannon, W J Floyd, W R Parry, Introductory notes on Richard Thompson's groups, Enseign. Math. (2) 42 (1996) 215-256 MR1426438

[11] F Degenhardt, Endlichkeitseigeinschaften gewiser Gruppen von Zöpfen unendlicher Ordnung, $\mathrm{PhD}$ thesis, Frankfurt (2000)

[12] P Dehornoy, Geometric presentations for Thompson's groups, J. Pure Appl. Algebra 203 (2005) 1-44 MR2176650

[13] P Dehornoy, The group of parenthesized braids, Adv. Math. 205 (2006) 354-409 MR2258261 
[14] P Dehornoy, I Dynnikov, D Rolfsen, B Wiest, Why are braids orderable?, Panoramas et Synthèses 14, Société Mathématique de France, Paris (2002) MR1988550

[15] I A Dynnikov, Three-page representation of links, Uspekhi Mat. Nauk 53 (1998) 237238 MR1691196

[16] I A Dynnikov, Recognition algorithms in knot theory, Uspekhi Mat. Nauk 58 (2003) 45-92 MR2054090

[17] D B A Epstein, J W Cannon, D F Holt, S V F Levy, M S Paterson, W P Thurston, Word processing in groups, Jones and Bartlett Publishers, Boston (1992) MR1161694

[18] D S Farley, Finiteness and CAT(0) properties of diagram groups, Topology 42 (2003) 1065-1082 MR1978047

[19] V Fock, Dual Teichmüller spaces arXiv:dg-ga/9702018

[20] L Funar, R Gelca, On the groupoid of transformations of rigid structures on surfaces, J. Math. Sci. Univ. Tokyo 6 (1999) 599-646 MR1742596

[21] L Funar, C Kapoudjian, The Ptolemy-Thompson group is asynchronously combable arXiv:math.GT/0602490

[22] L Funar, C Kapoudjian, On a universal mapping class group of genus zero, Geom. Funct. Anal. 14 (2004) 965-1012 MR2105950

[23] S M Gersten, H Short, Small cancellation theory and automatic groups. II, Invent. Math. 105 (1991) 641-662 MR1117155

[24] É Ghys, V Sergiescu, Sur un groupe remarquable de difféomorphismes du cercle, Comment. Math. Helv. 62 (1987) 185-239 MR896095

[25] P Greenberg, V Sergiescu, An acyclic extension of the braid group, Comment. Math. Helv. 66 (1991) 109-138 MR1090167

[26] R I Grigorchuk, V V Nekrashevich, V I Sushchanskiü, Automata, dynamical systems, and groups, Tr. Mat. Inst. Steklova 231 (2000) 134-214 MR1841755

[27] V Guba, M Sapir, Diagram groups, Mem. Amer. Math. Soc. 130 (1997) viii+117 MR1396957

[28] C Kapoudjian, V Sergiescu, An extension of the Burau representation to a mapping class group associated to Thompson's group T, from: "Geometry and dynamics", Contemp. Math. 389, Amer. Math. Soc., Providence, RI (2005) 141-164 MR2181963

[29] M Kontsevich, Y Soibelman, Affine structures and non-archimedean spaces arXiv: math. AG/0406564

[30] P Lochak, L Schneps, The universal Ptolemy-Teichmüller groupoid, from: "Geometric Galois actions, 2”, London Math. Soc. Lecture Note Ser. 243, Cambridge Univ. Press, Cambridge (1997) 325-347 MR1653018 
[31] A V Malyutin, Fast algorithms for the recognition and comparison of braids, Zap. Nauchn. Sem. S.-Peterburg. Otdel. Mat. Inst. Steklov. (POMI) 279 (2001) 197-217, 250 MR1846081

[32] D R Mason, On the 2-generation of certain finitely presented infinite simple groups, J. London Math. Soc. (2) 16 (1977) 229-231 MR0466324

[33] J Matthews, The conjugacy problem in wreath products and free metabelian groups, Trans. Amer. Math. Soc. 121 (1966) 329-339 MR0193130

[34] L Mosher, Mapping class groups are automatic, Ann. of Math. (2) 142 (1995) 303-384 MR1343324

[35] R C Penner, The universal Ptolemy group and its completions, from: "Geometric Galois actions, 2", London Math. Soc. Lecture Note Ser. 243, Cambridge Univ. Press, Cambridge (1997) 293-312 MR1653016

[36] R C Penner, J L Harer, Combinatorics of train tracks, Annals of Mathematics Studies 125, Princeton University Press, Princeton, NJ (1992) MR1144770

[37] D J S Robinson, A course in the theory of groups, second edition, Graduate Texts in Mathematics 80, Springer, New York (1996) MR1357169

[38] V Sergiescu, Graphes planaires et présentations des groupes de tresses, Math. Z. 214 (1993) 477-490 MR1245207

[39] B Wiest, Diagram groups, braid groups, and orderability, J. Knot Theory Ramifications 12 (2003) 321-332 MR1983088

[40] D Witte, Arithmetic groups of higher Q-rank cannot act on 1-manifolds, Proc. Amer. Math. Soc. 122 (1994) 333-340 MR1198459

Institut Fourier BP 74, UMR 5582, University of Grenoble I

38402 Saint-Martin-d'Hères cedex, France

Laboratoire Emile Picard, UMR 5580, University of Toulouse III

31062 Toulouse cedex 4, France

funar@fourier.ujf-grenoble.fr, ckapoudj@picard.ups-tlse.fr

http://www-fourier.ujf-grenoble.fr/ funar/

Proposed: Shigeyuki Morita

Seconded: Joan Birman, Jean-Pierre Otal
Received: 26 June 2007

Accepted: 21 November 2007 